\title{
Sample handling in surface sensitive chemical and biological sensing: a practical review of basic fluidics and analyte transport
}

\author{
Norbert Orgovan $^{\mathbf{a}, \mathbf{b}}$, Daniel Patko ${ }^{\mathbf{b}, \mathbf{c}}$, Csaba Hos $^{\mathrm{d}}$, Sándor Kurunczi ${ }^{\mathbf{b}}$, Bálint Szabó ${ }^{\mathbf{a}}$ \\ Jeremy J. Ramsden ${ }^{\mathbf{e}, \mathbf{f}}$ and Robert Horvath ${ }^{\mathbf{b}}$
}

${ }^{a}$ Department of Biological Physics, Eötvös University, Pázmány P. sétány 1A, H-1117 Budapest, Hungary

${ }^{\mathbf{b}}$ Department of Photonics, Institute for Technical Physics and Materials Science, Research Centre for Natural Sciences (MTA TTK MFA), H-1121, Konkoly-Thege Miklós út 29-33,

\section{Budapest, Hungary}

${ }^{\mathbf{c}}$ Doctoral School of Molecular- and Nanotechnologies, Faculty of Information Technology, University of Pannonia; H-8200 Egyetem u.10, Veszprém, Hungary

${ }^{\mathrm{d}}$ Department of Hydraulic Machines, Budapest University of Technology and Economics (BME), 1521 Budapest, Pf. 91, Hungary ${ }^{\mathrm{e}}$ Clore Laboratory, University of Buckingham, MK18 1EG, UK

${ }^{\mathbf{f}}$ Centre for Molecular Recognition, Collegium Basilea (Institute of Advanced Study), Hochstrasse 51. CH-4053 Basel

email: horvathr@mfa.kfki.hu 


\section{Contents}

2

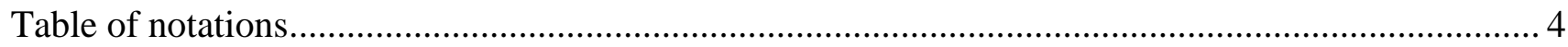

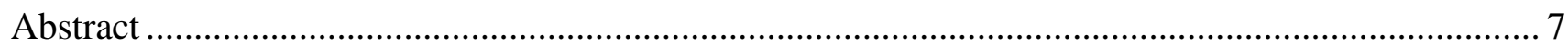

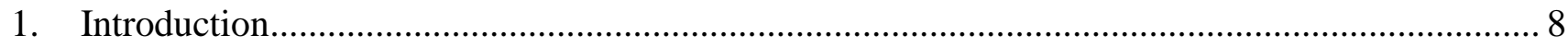

2. Short overview of the basic theory of fluidics and analyte transport ..................................... 10

2.1 Fluid handling in cuvettes and cylindrical connecting tubes - flow rates............................... 10

2.2 Diffusion of analytes: the diffusion boundary layer.......................................................... 12

2.3 Transport to, adsorption on, and desorption from the sensor surface .................................. 14

2.3.1 Adsorption model for single-component solutions.................................................... 14

2.3.2 Desorption from the sensor surface - readsorption and rebinding ................................. 16

2.3.3 Adsorption from complex, multicomponent analyte solutions....................................... 17

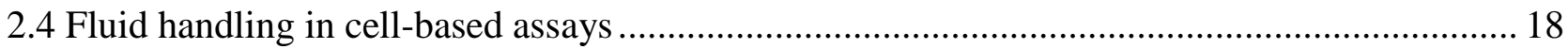

2.4.1 Adhesion assays: general considerations, and cell deposition in an environment without

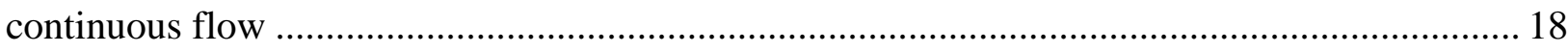

2.4.2 Mechanotransduction assays: cells in a flow environment............................................ 20

2.4.3 Signaling assays: the challenge of compound addition to pre-attached cells .................... 22

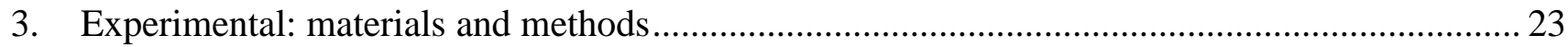

3.1 Optical waveguide lightmode spectroscopy …................................................................ 23

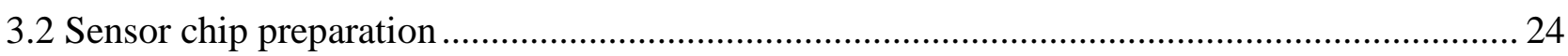

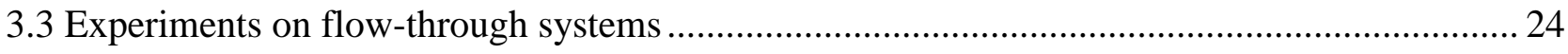

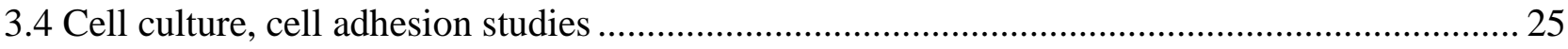

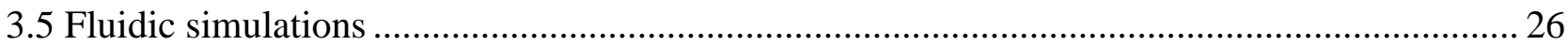

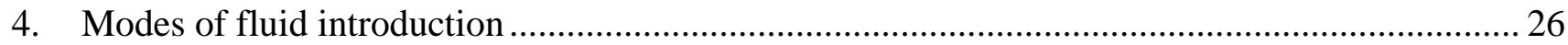

4.1 Closed cuvette without flow and manual fluid introduction using a pipette: monitoring cell

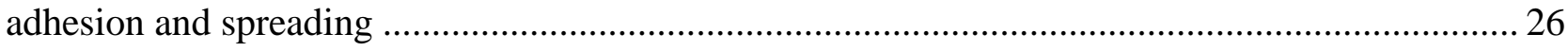

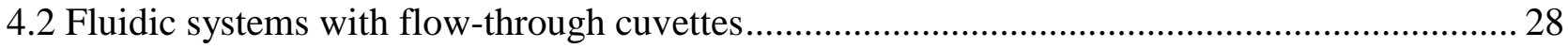

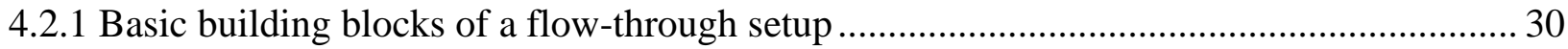

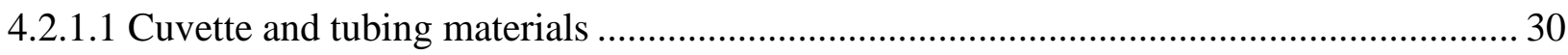

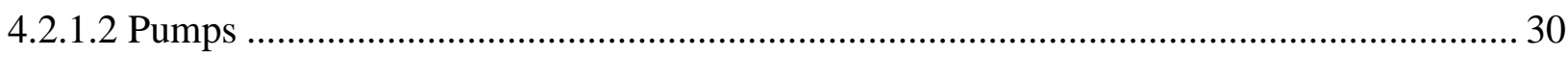

4.2.2 Exchange of samples using flow-through fluidics.................................................... 31

4.2.2.1 Diffusion due to the dead volumes of the cuvette .................................................... 31 
4.2.2.2 Length of tubing 33

4.3 Injection systems for the introduction of limited amount of sample...................................... 34

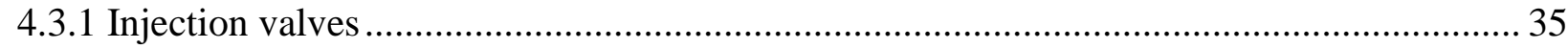

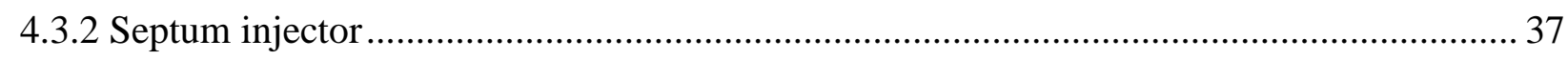

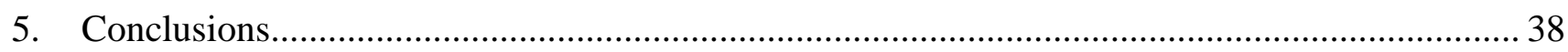

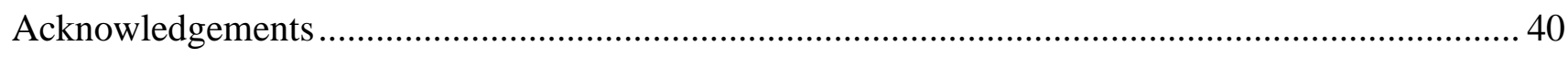

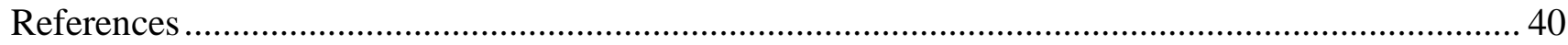




\section{Table of notations}

\begin{tabular}{|c|c|}
\hline Symbol & Meaning \\
\hline$A$ & cross section of a tube \\
\hline$\alpha$ & constant of proportionality between $N$ and analyte concentration \\
\hline$C$ & constant characterizing the geometry of a cuvette \\
\hline$c_{0}$ & bulk concentration \\
\hline$c_{\mathrm{v}}$ & analyte concentration in the diffusion boundary layer \\
\hline$\Gamma_{\mathrm{r}}$ & surface mass density of reversibly adsorbed molecules \\
\hline$\Gamma_{\mathrm{i}}$ & surface mass density of irreversibly adsorbed molecules \\
\hline$d$ & diameter of a tube \\
\hline$D$ & diffusion coefficient \\
\hline$\delta$ & thickness of the diffusion boundary layer \\
\hline$F$ & volumetric flow rate \\
\hline$\Phi$ & available area function \\
\hline$g$ & gravitational constant \\
\hline$k_{\mathrm{B}}$ & Boltzmann constant \\
\hline$k_{\mathrm{d}}$ & first order rate coefficient of desorption \\
\hline$k_{\mathrm{i}}$ & rate coefficient of irreversible adsorption \\
\hline$k_{r}$ & rate coefficient of reversible adsorption \\
\hline$L$ & length of tubing \\
\hline
\end{tabular}




\begin{tabular}{|c|c|}
\hline$N$ & effective refractive index \\
\hline$\mu$ & dynamic viscosity \\
\hline$n_{\mathrm{C}}$ & refractive index of the cover layer (medium) \\
\hline$p_{1}, p_{2}$ & pressures at the two ends of a tubing \\
\hline$q$ & distance between the in-and outlet apertures of a flow-through cuvette \\
\hline$r$ & radius of a diffusing object \\
\hline$R$ & inner radius of a tube \\
\hline $\operatorname{Re}$ & Reynolds number \\
\hline$\rho$ & density of a fluid \\
\hline$\rho_{\mathrm{c}}$ & mean density of a mammalian cell \\
\hline$\rho_{\mathrm{m}}$ & density of an aqueous medium \\
\hline$S$ & unit area \\
\hline$t$ & time \\
\hline$t_{\mathrm{s}}$ & time required to saturate a biosensor signal \\
\hline$T$ & absolute temperature \\
\hline$\tau_{\mathrm{w}}$ & wall shear stress \\
\hline$v$ & mean velocity of a fluid \\
\hline$v_{\mathrm{fd}}$ & velocity of cells during forced deposition \\
\hline$v_{0}$ & velocity of the leading edge \\
\hline$v_{\text {term }}$ & terminal velocity of a cell during sedimentation \\
\hline$V$ & unit volume \\
\hline
\end{tabular}




\begin{tabular}{cc}
\hline$V_{\mathrm{T}}$ & total sample volume of a cuvette \\
\hline$V_{\mathrm{C}}$ & volume of the convective zone of a cuvette \\
\hline$V_{\mathrm{D}}$ & volume of the unflushed regions in a cuvette \\
\hline$x$ & distance \\
\hline$z$ & distance between the point of inlet and point of sensing in a cuvette \\
\hline
\end{tabular}




\section{Abstract}

This paper gives an overview of the advantages and associated caveats of the most common sample handling methods in surface-sensitive chemical and biological sensing. We summarize the basic theoretical and practical considerations one faces when designing and assembling the fluidic part of the sensor devices. The influence of analyte size, the use of closed and flow-through cuvettes, the importance of flow rate, tubing length and diameter, bubble traps, pressure-driven pumping, cuvette dead volumes, and sample injection systems are all discussed. Typical application areas of particular arrangements are also highlighted, such as the monitoring of cellular adhesion, biomolecule adsorption-desorption and ligand-receptor affinity binding. Our work is a practical review in the sense that for every sample handling arrangement considered we present our own experimental data and critically review our experience with the given arrangement. In the experimental part we focus on sample handling in optical waveguide lightmode spectroscopy (OWLS) measurements, but the present study is equally applicable for other biosensing technologies in which an analyte in solution is captured at a surface and its presence is monitored. Explicit attention is given to features that are expected to play an increasingly decisive role in determining the reliability of (bio)chemical sensing measurements, such as analyte transport to the sensor surface; the distorting influence of dead volumes in the fluidic system; and the appropriate sample handling of cell suspensions (e.g. their quasi-simultaneous deposition). At the appropriate places, biological aspects closely related to fluidics (e.g. cellular mechanotransduction, competitive adsorption, blood flow in veins) are also discussed and compared to their models used in biosensing.

\section{Keywords:}

fluidic systems

dead volumes

optical biosensors analyte transport

label-free detection

fluid handling of live cells 


\section{Introduction}

Nowadays, the field of chemical and biological sensing goes well beyond the application of novel technologies to detect the presence of a given analyte. The range of table-top biosensor instrumentation is wide and their use helps many laboratories worldwide to answer important scientific and technological questions in biomaterials and surface sciences, drug development, biophysics, (bio)nanotechnology and cell biology [1], as well as supramolecular chemistry [2].

Biosensing technologies enabling label-free detection at a solid-liquid interface include surface plasmon resonance (SPR) (and SPR imaging, SPRi) [3,4,5], quartz crystal microbalance (QCM) [6], dual polarization interferometry (DPI) [7,8], grating-coupled interferometry (GCI) [9, 10,11,12], the high-throughput compatible resonant waveguide grating biosensing $[13,14,15]$, and optical waveguide lightmode spectroscopy (OWLS) $[8,16,17,18]$. OWLS instruments are currently among the most sensitive commercial label-free biosensors which enable the monitoring of various processes accompanied by refractive index changes 100-200 nm above a sensor surface . OWLS permits real-time, high-sensitivity (adsorbed mass coverages as low as $10 \mathrm{pg} / \mathrm{mm}^{2}$ can be detected) label-free detection with a typical temporal resolution of 10-20 s, which makes it an ideal research tool in several fields, including the online monitoring of protein adsorption $[19,20,21,22]$, bacterial [23,24] and mammalian cell adhesion [25,26,27,28,29,30], supported lipid-bilayer deposition [31,32], polyelectrolyte multilayer build-up [33,34,35], nanoparticle surface adhesion and assembly [36,37], and receptor-ligand interactions [38,39].

An outstanding advantage of surface-sensitive label-free biosensing technologies is that they allow so-called "real-time monitoring", thus generating kinetic data. Kinetic modeling based on such valuable data enables the molecular or cellular process under investigation to be quantitatively characterized in detail. For example, adsorption and desorption rates at model surfaces, or kinetic rates in affinity binding can be determined [40,41], which contribute to a better understanding of the mechanism underlying the investigated surface process. Kinetic analysis of experimental data is 
furthermore the key to a detailed characterization of cell adhesion [14], including the separation of the governing cellular processes from less relevant ones, and the quantification of the differences arising from different cell types, substrata, media and other environmental factors $[14,42,43,44,45,46]$. However, kinetic data is often misinterpreted. A common reason is that the temporal evolution of the concentration distribution of the investigated objects over the sensing area is not known, or is uncontrolled, which is closely related to the applied fluidic system.

In the majority of applications, biomolecules or cells are investigated in aqueous solutions mimicking their native environment. OWLS measurements are usually carried out by sealing a cuvette containing the medium over the sensing area. Generating flow to ensure a continuous supply of fresh analyte, changing solutions, executing washing steps, etc. all require some kind of fluid handling system.

A variety of fluidic systems can be built from the wide range of commercially available accessories in order to optimize sample handling for a particular application. The simplest systems use a plain well without flow (henceforth "closed cuvette"). However, this simplest configuration may yield less informative data as compared to measurements when the liquid sample is continuously flowed over the sensing area [17]. More sophisticated fluidic systems, therefore, consist of pumps, sections of tubes of different lengths and diameters, the junctions between them, and a flow-through cuvette (Fig. 1). Other components such as bubble traps can be integrated as well [47].

However, in several applications, especially when working with costly analytes, the continuous flow of fresh material is not feasible. In these applications, some kind of sample injection system has to be used, preferably in combination with a flow-through system. This facilitates sample handling automation: the sample is introduced by aspiration or injection and subsequently guided to the cuvette. This technique is called flow injection analysis (FIA), which has many valuable features for on-line biosensor measurements [48]. Although FIA can be used to modify the sample matrix or for preconcentration or derivatization, biosensing has predominantly exploited the precise and reproducible fluid handling capability of the injection valves used with FIA [49,50]. 
Importantly, the fluidic system is a critical part of any biosensor setup, as it is the most common source of experimental errors, which endanger the reliability of the recorded data and may lead to serious misinterpretations. This is especially true when elaborate manipulations with the samples have to be made or when the kinetics of the processes are deeply interpreted. The present work overviews the most important theoretical and practical considerations involved in the design of a fluidic system, with the aim of facilitating finding the best arrangement for a given application; and emphasizing how to obtain reliable data, which can be then subjected to detailed analysis. In the experimental part we focus on sample handling in OWLS measurements, but this study is equally applicable for other biosensing technologies in which an analyte in solution is captured at a surface and its presence is monitored. At the appropriate places, key biological phenomena closely related to fluidics (e.g. cellular mechanotransduction, competitive adsorption, blood flow in veins) are also highlighted to indicate how in vivo conditions can be better approximated and understood. After a brief description, the reader is referred to papers specifically involved in these problems.

\section{Short overview of the basic theory of fluidics and analyte transport}

\subsection{Fluid handling in cuvettes and cylindrical connecting tubes - flow rates}

The supply of analyte solution is usually pumped through tubes to reach a tube-like cuvette placed over the sensing area. The nature of the flow is determined by the value of the dimensionless Reynolds number (Re), defined as the ratio of inertial forces (due to the flow) and viscous forces (due to the internal friction of the fluid):

$$
R e=\frac{\rho v^{2} / d}{\mu v / d^{2}}=\frac{\rho v d}{\mu}=\frac{\rho F d}{\mu A}
$$

where $\rho, \mu$, and $v$ are the density, dynamic viscosity and mean velocity of the fluid, respectively. $d$ is the diameter of the tube, $F$ is the volumetric flow rate and $A$ is the cross-section of the tube [51]. 
Below a critical value of $R e$, viscous forces dominate and the flow is laminar, i.e. the velocity depends only on the perpendicular distance from the fore-axis of the tube. In this case the evolving streamlines are parallel to the axis, and the stream volume can be split into coaxial layers in which the flow rates are the same (Fig. 1). The flow is surely laminar in a tube when $\operatorname{Re}<2300$, and turbulent when $R e>4000$. In typical biosensing experiments the flow rates guarantee that $R e$ is well below the former threshold, therefore only laminar flow occurs [52]. With the present focus on instrumental miniaturization (e.g., the creation of labs-on-chips) internal tubing diameter can be as small as $100 \mu \mathrm{m}$ or even less; $R e$ is then of order unity [53]. An important exception to the preponderance of laminar flow is when an atypically high flow rate is used in order to effectively flush the dead volumes of the cuvette by generating turbulence (see $\S 2.5 .3$ and $\S 4.3 .2$ ).

A number of practical aspects set upper and lower limits to the actual flow rate with which an analyte solution can be pumped during a biosensing experiment. The chosen rate determines how much sample is needed for one measurement and whether the investigated process will be kinetically or transport-limited (see $\$ 2.3$ ); in the latter case the resulting kinetic data will not be representative of the true surface process (as may happen in Biacore experiments using the popular dextran-coated surfaces [54]).

Expense or limited availability of a sample pushes one to use the least possible amount of sample in a measurement and, as an initial strategy, the amount can easily be decreased using lower flow rates. Additionally, it has been claimed that a lower detection limit can be achieved by using low flow rates [55]. However, diminishing the flow rate will ultimately result in transport-limited adsorption of analyte, during which the surface adsorption step in the sensing area is fast compared to the rate the analyte is supplied by the flow system and, therefore, the true surface process will not dominate the kinetics of the obtained signal (see $\S 2.3$ ), which is presumably what one wishes to quantify. Moreover, during data evaluation it is a common assumption that by the time presence of the sample first appears in the recorded data, the cuvette has already been perfectly flushed 
through by the sample solution (e.g., the liquids have been perfectly exchanged). The lower the flow rate, the more carefully this assumption needs scrutiny $(\S 2.3)$.

In this review we consider only pressure-generated flow. If it is laminar, the Hagen-Poiseuille law states that

$$
p_{1}-p_{2}=\frac{8 \mu L}{\pi R^{4}} F,
$$

where $p_{1}$ and $p_{2}$ are the pressures at the two ends of the tube, and $R$ and $L$ are the inner radius and length of the tube, respectively. Based on the formal similarity of Eq. 2 to Ohm's law, the multiparameter factor multiplying $F$ on the right side of Eq. 2 is usually referred to as the "resistance" of the tube. Note how this factor depends on $R$ and $L$. If this resistance is changed following the modification of the setup, it is strongly recommended to check whether the pump is powerful enough to generate the same flow rate as earlier.

Fluid dynamics and flow in a tube have important biological aspects, especially when considering flow in blood vessels. Note, however, that pulsatile flow of (the non-Newtonian) blood in the compliant vessels is far too complex to be characterized by the classical Poiseuille fluid dynamics (describing steady flow in a rigid tube of circular cross-section) [56] briefly introduced above.

\subsection{Diffusion of analytes: the diffusion boundary layer}

Surface sensitive, high-performance label-free techniques require the sample of interest to be brought into close contact with the surface of the sensor (the planar waveguide in OWLS, RWG or GCI or the gold surface in SPR). During analyte transport two fundamental processes have to be distinguished: convection (sometimes called advection) and diffusion. The liquids in which the biological or chemical objects under investigation are dissolved or suspended are usually introduced above the sensor surface by pumps, engendering convection [51]. At the solid-liquid interface, however, the tangential flux is always zero and, therefore, another transport process, diffusion, 
dominates close to the surface. The importance of diffusion relative to other transport phenomena (convection, sedimentation) is strongly dependent on the nature of the suspended analyte objects: their size, buoyancy, 3D shape and structure are all important factors.

An object having a diffusion coefficient $D$ will diffuse to an expected distance of

$$
\left\langle x^{2}\right\rangle^{1 / 2}=\sqrt{q_{\mathrm{i}} D t}
$$

from its starting point during elapsed time $t . q_{\mathrm{i}}$ is a numerical constant which depends on dimensionality; it is 2,4 or 6 for 1,2 , or 3 dimensional diffusion, respectively. Assuming spherical symmetry for the analyte object, the Stokes-Einstein equation can be used to calculate $D$ :

$$
D=k_{\mathrm{B}} T / 6 \pi \mu r
$$

where $k_{\mathrm{B}}$ denotes the Boltzmann constant, $T$ the absolute temperature, and $r$ the radius of the object; if it is small the effect of gravity is negligible.

Diffusion plays an important role in other parts of the fluidic system as well as above the sensor surface. For example, due to diffusion, the flow front in a tube is smeared out in the direction of its fore-axis, although to a minor extent compared to that caused by diffusion perpendicular to the axis. To understand the importance of the latter, one has to be familiar with the concept of the diffusion boundary layer.

As the net result of friction between the fluid and the wall of the tubing, the laminar flow rate profile is parabolic inside the tubing and the cuvette (cf. Eq. 2) (Fig. 1). In the vicinity of the walls convection is negligible relative to diffusion (i.e. the dimensionless Péclet number, defined as the ratio of advective transport rate to the diffusive transport rate [57], is $P e=2 v R / D \ll 1$ ), but the former dominates further away from the surface (i.e. Pe 》1). The zone in which diffusion is the dominant process is called the diffusion boundary layer; it has a thickness of $[17,41,51]$ : 


$$
\delta=\left(\frac{3}{2}\right)^{2 / 3}\left(\frac{D \cdot C}{v_{0}}\right)^{1 / 3}
$$

where $v_{0}$ is the velocity of the leading point. $\delta$ is dependent on the geometry of the fluidic system mainly through the constant $C$. For example, in the case of a cylindrical cuvette with radius $R$, $C=z R$, where $z$ is the distance between the inlet of the cuvette and the point of sensing. It can be calculated from Eq. 4 that under typical experimental conditions $\delta$ is $1-100 \mu \mathrm{m}$.

\subsection{Transport to, adsorption on, and desorption from the sensor surface}

Surface-sensitive optical biosensors detect refractive index changes close to a solid-liquid interface (§3.1). Since the adsorption of the investigated objects (molecules, viruses, bacteria, vesicles, etc.) onto the sensor and their desorption from it can strongly affect the refractive index in the sensing zone, an adsorption model has to be considered for a complete description of detectable refractive index changes.

\subsubsection{Adsorption model for single-component solutions}

The simplest kinetic adsorption model of practical use distinguishes reversible (subscript $r$ ) and irreversible (subscript i) adsorption from a solution containing only a single type of analyte. To a first approximation the evolution of the surface excess of the reversibly and irreversibly adsorbing particles can be given by the following set of differential equations

$$
\frac{\mathrm{d} \Gamma_{\mathrm{r}}}{\mathrm{d} t}=k_{\mathrm{r}} c_{\mathrm{v}} \Phi(\Gamma)-k_{\mathrm{d}} \Gamma_{\mathrm{r}} \quad \text { and } \quad \frac{\mathrm{d} \Gamma_{\mathrm{i}}}{\mathrm{d} t}=k_{\mathrm{i}} c_{\mathrm{v}} \Phi(\Gamma)
$$

where $\Phi$ is the available area function characterizing the free surface area available for the adsorbing objects. Here, $k_{\mathrm{r}}$ and $k_{\mathrm{i}}$ are the rate coefficients for reversible and irreversible adsorption, respectively, while $k_{\mathrm{d}}$ is the first order rate coefficient for desorption; their actual values are 
characteristic of both the adsorbing object and the surface. The concentration of adsorbing particles in the diffusion boundary layer is denoted as $c_{v}$ (note, that the layer probed by the sensor is generally much more thinner than the diffusion boundary layer). The above model assumes that the reversibly and irreversibly adsorbing particles have the same size, shape, and surface affinity and that they randomly compete with each other. For example, a spherical, but chemically inhomogeneous protein could adsorb reversibly in certain orientations, otherwise irreversibly; the probabilities of the different orientations are subsumed in the rate coefficients. The total mass at the surface is the sum of reversibly and irreversibly adsorbed molecules, $\Gamma=\Gamma_{\mathrm{r}}+\Gamma_{\mathrm{i}}$. Note that $\Phi(\Gamma)=1$ if $\Gamma=0$, and $\Phi(\Gamma)=0$ if the whole surface is covered to an extent that there is not enough free space for further adsorbing objects. The available area function depends on the shape of the adsorbing object [58], and usually has a complicated polynomial dependence on the occupied surface area. Note, that in the special case, when relatively large receptors are deposited on the sensor surface and used to capture a smaller analyte, the available area function has a simple linear form (Langmuir adsorption, [59]).

A major aim of kinetic data analysis is the determination of the rate coefficients, which can be used to elucidate the molecular mechanism of the adsorption process. A key issue here is the appropriate characterization of the temporal evolution of $c_{v}$, which is not always the same as that of the bulk concentration $c_{0}$.

The adsorption model described by Eq. 6 predicts that $\mathrm{d} I / \mathrm{d} t$ obtains its maximum value at the beginning of the adsorption process $(\Phi=1)$ and monotonically decreases as the adsorption proceeds (Fig. 2 dashed line). In contrast, experimental data typically reveals a different behavior: at the initial stage of the adsorption, a transient regime can be observed. The process is said to be transport-limited if the adsorption of the particle of interest is faster than the replenishment of material in the vicinity of the surface [60]. As adsorption proceeds, more of the surface is occupied by adsorbed molecules leaving less space available for newly arriving ones, resulting in a slower rate 
of surface mass increase. In this so-called kinetically limited regime the rate of adsorption, $\mathrm{d} \Gamma / \mathrm{d} t$ decreases monotonically with time, reflecting the diminishing available area.

During transport-limited adsorption, $c_{\mathrm{v}}$ is not equal to $c_{0}$; differences are the net result of diffusion from the bulk to the probed layer, and adsorption to and desorption from the surface. These can be taken into account by the following equation [41]:

$$
V \frac{\mathrm{d} c_{\mathrm{v}}}{\mathrm{d} t}=S\left(D \frac{c_{0}-c_{\mathrm{v}}}{\delta}-\left(k_{\mathrm{r}}+k_{\mathrm{i}}\right) c_{\mathrm{v}} \Phi+k_{\mathrm{d}} \Gamma_{\mathrm{r}}\right)
$$

where $V$ and $S$ are unit volume and area, respectively. In a flow-through cuvette $c_{0}$ is kept constant above the diffusion boundary layer by supplying a continuous flow of analyte. If, however, flushing the volume above the diffusion boundary layer is not effectively instantaneous (compared to the sampling rate of the instrument and the duration of the whole adsorption process), a transient regime might appear in the kinetics of adsorption. As a result of this so-called flushing effect, $c_{0}$ and, therefore, $c_{v}$ are ill-defined in the early stages of solution exchange. The flushing effect can be aggravated by diffusion to and from the dead volumes - those volumes that are not part of the usergenerated laminar flow and which, therefore, cannot be completely and effectively instantaneously flushed. Dead volumes act like reservoirs when a sample is exchanged in the cuvette and, therefore, initialize diffusion-driven changes in the local sample concentration; this essentially effects the OWLS signal, which will be considered later (§4.2.2).

\subsubsection{Desorption from the sensor surface - readsorption and rebinding}

If adsorption is not irreversible, or to test whether it is, the adsorption stage with analyte in the fluidic system is followed by a washing/flushing stage when analyte-free medium (e.g. pure buffer) is introduced into the cuvette. During this stage $c_{0}$ (Eqn. 7) becomes zero and, typically desorption dominates (i.e. the term with $k_{\mathrm{d}}$ becomes the most significant). It is important to note that $c_{\mathrm{v}}$ is not 
zero except near the end of the stage. Readsorption of freshly desorbed material occurs and is fully taken into account by using the same equations (Eqn. 6 and 7) for fitting the desorption part of the kinetics as for the adsorption part; the only difference is that $c_{0}$ is set to zero (exactly how this is done depends on consideration of the dead volumes, see $\S 4.2 .2 .1)$.

It has to be noted that the above effect is also important in bioaffinity measurements, when receptors are immobilized on the sensor surface and ligands in solution are captured; if diffusion is slow compared to the on-rate (i.e. the dissociation phase is transport limited), a considerable amount of ligand will rebind rather than diffusing into the bulk solution $[60,61]$. If this rebinding effect is not taken into account during data analysis, an off-rate that is much lower than the actual one will be obtained [59]. The transport-limited dissociation phase under flow conditions can be described, to a good approximation, with the special analytical formula developed by Schuck and Minton [62]. Another alternative possibility is to prevent rebinding by adding competing ligand during the dissociation phase [59].

\subsubsection{Adsorption from complex, multicomponent analyte solutions}

Importantly, the description of surface adsorption given in $§ 2.3 .1-2$ can be applied for singlecomponent solutions only, and cannot be straightforwardly generalized for adsorption from a complex, multicomponent analyte solution. Adsorption from a complex sample takes place in a sequential and competitive manner, so the composition of the adsorbed layer is constantly evolving (Vroman effect) $[63,64,65,66]$. Those proteins which are bigger and have more affinity to the surface eventually displace the ones that occupy the surface areas more rapidly. As a result, for example the maximal ratio of the surface occupied by fibrinogen is generally obtained at intermediate blood serum concentrations [64]. 


\subsection{Fluid handling in cell-based assays}

Surface-sensitive biosensors enable various activities of adherent cells to be monitored with high sensitivity and high temporal resolution [14,30,67,68]. Cell-based studies performed on labelfree biosensors can basically be classified as adhesion, signaling, or mechanotransduction assays, each of which requires different sample handling strategies. In general, sample handling in cellbased assays remains always challenging. Additional difficulties of sample handling as compared to biochemical assays comes from that fact, that living cells are highly dynamic entities very sensitively responding to changes in their surroundings, and are also able to actively modify their environment (e.g through metabolism or secretion). Therefore, several criteria regarding sample handling have to be satisfied if one wishes to reliably monitor the behavior of living cells using OWLS or other surface-sensitive biosensors.

2.4.1 Adhesion assays: general considerations, and cell deposition in an environment without continuous flow

In vivo, cell adhesion and spreading are induced by amino acid sequences presented by proteins of the extracellular matrix (ECM), which are specifically recognized by the adhesion receptors of the cell $[69,70]$. Once the connection between some adhesion receptors and ligands are established, the cell will begin to spread by actively reorganizing its cytoskeleton; this attachment is essential for most cells to survive and properly function. In most cases, therefore, the sensor should be pre-coated with either a synthetic (e.g. PLL-g-PEG-RGD [14,71]) or a purified (e.g. collagen, fibrinogen) polymer mimicking the ECM in order to specifically promote the spreading of the subsequently seeded cells. 
In cell spreading assays, the molecular coating should ideally be able to specifically activate the adhesion receptors and retract soluble biomolecules at the same time; so any change in the signal will originate from the spreading of cells, and the adsorption of biomolecules secreted by the cells will not significantly contribute to it $[14,71]$. (Noted, that kinetic readout of multiple parameters (the position and width of the resonance peaks) in OWLS enables to distinguish the refractive index variations caused by secreted molecules from refractive index changes provoked by cellular spreading $[29,30,72]$. However, this is a unique feature, and not common to all surface-sensitive biosensors.) The biosensor baseline is established with assay buffer (physiological buffer, in which the cells will be eventually bathed) above the pre-coated sensors. When seeding cells, a number of practical considerations should be taken into account. First, the introduction and deposition of the cells should ideally result in their uniform distribution on the sensor surface. Second, a sensing area coverage of somewhat more than 50\% (typically meaning 2000-6000 deposited eukaryotic cells $/ \mathrm{mm}^{2}$ ) is necessary to allow the relative adhesion strength to be determined; in OWLS-based cytometry, the refractive index change in the evanescent field at 50\% coverage is a quantitative measure of cellsubstratum adhesivity $[46,67,73]$. However, there is an upper limit to the desirable cell density; in order to allow uniformly distributed cells to have enough space for all of them to spread the formation of multilayers or that of cell aggregates should be avoided. Third, the duration of the deposition should be as short as possible; if there is significant cell-to-cell variation in the deposition time it is difficult to distinguish between signals corresponding to deposition and to the active process of spreading, since while some cells are still in suspension, others already on the surface will have begun to spread. One can try to separate the signals corresponding to the two processes via data processing - a number of numerical and theoretical models aim at describing the deposition kinetics of spherical particles sedimenting under the combined influence of Brownian collisions with the molecules of the bathing medium and the gravitational force [74,75]. However, it is more straightforward to achieve a quasi-instantaneous (compared to the time required for initiation of active spreading) deposition of cells. Cells in solution can be considered as spherical objects to an 
excellent approximation. Let us first assume them to have zero initial velocity. If they fall in a viscous fluid by their own weight, then terminal velocity is reached when the frictional force combined with the buoyant force exactly balances the gravitational force. The resulting terminal or settling velocity is given by [76,77]

$$
v_{\text {term }}=\frac{2}{9} \frac{\left(\rho_{\mathrm{c}}-\rho_{\mathrm{m}}\right) g}{\mu} r^{2}
$$

The dynamic viscosity and the density of the aqueous medium can usually be assumed to be the same as that of the water, i.e. $\mu=6.92 \times 10^{-3} \mathrm{~g} /(\mathrm{cm} \times \mathrm{s})$ and $\rho_{\mathrm{m}}=0.9933 \mathrm{~g} / \mathrm{cm}^{3}$ at $T=37^{\circ} \mathrm{C}$ respectively; the radius of a typical mammalian cell is $r=7 \mu \mathrm{m}$ [78], its density is $\rho_{\mathrm{c}}=1.1 \mathrm{~g} / \mathrm{cm}^{3}$ [77]; and the gravitational constant is $g=981 \mathrm{~cm} / \mathrm{s}^{2}$. This yields a terminal velocity of $v_{\text {term }}=0.0016 \frac{\mathrm{cm}}{\mathrm{s}}=16 \mu \mathrm{m} / \mathrm{s}$, which is so slow that concerns about the achievability of quasiinstantaneous deposition of all cells can be rightfully raised. However, cells can be made to move with velocity $v_{\mathrm{fd}}$ during their introduction into the cuvette if the pipette with which they are introduced is oriented perpendicular to the sensor surface (forced deposition). Supposing that a volume of $0.1 \mathrm{ml}$ suspension is run through the end of a cylindrical pipette tip having a diameter of $0.5 \mathrm{~mm}$ in a time interval of $5 \mathrm{~s}$ : then $v_{\mathrm{fd}}=10.2 \mathrm{~cm} / \mathrm{s}$; which is sufficiently high to result in the desired quasi-instantaneous deposition of all cells. This was confirmed experimentally ( $\$ 4.1)$, when we used the forced deposition technique to seed cells on the sensor surface, and then successfully monitored their subsequent spreading. Forced deposition can be also achieved by centrifugation [79].

\subsubsection{Mechanotransduction assays: cells in a flow environment}

Cells are responsive to mechanical stimuli; external forces influence the formation of adhesion sites, cell orientation, gene expression, and more [56,80]. To study the effects of external forces on cell behavior, cells are often investigated in a flow environment which approach may have more 
biological relevance as compared to experiments performed in the total absence of flow. The workflow of a standard mechanotransduction assay starts with a surface functionalization step to obtain a cell-adhesive surface; then cells are seeded (preferably in high densities), and allowed to establish the first contacts with the surface for a given time; and finally, a laminar flow with a welldefined rate is applied, which may be increased in a stepwise manner. However, increased scrutiny has to be given to the experimental design to avoid cell damage in such a study [81]. Cells in a flow environment may experience various forces; the most likely sources of cell injury are relatively large shear forces, interaction with small eddies occurring in turbulent flow, or interaction with bubbles during their breakup [81]; the latter two can be easily avoided in a biosensor experiment, but the first one needs further considerations. In vivo exposure of various cell types (including red blood cells, immune cells, tumor cells, endothelial cells) to flow predominantly occurs in the circulation system. Highest flow shear stresses are experienced in the arterial circulation, where time-averaged values are approximately in the range of $4-30$ dynes $\mathrm{cm}^{-2}$ [82]. In comparison, the wall shear stress (which is the maximum shear stress) of a Newtonian fluid during laminar flow in a straight cylindrical tube is [82]:

$$
\tau_{\mathrm{w}}=\frac{4 \mu F}{\pi R^{3}}
$$

In a typical biosensor experiment $R=0.0255 \mathrm{~cm}$, and $F=0.01 \mathrm{~cm}^{3} / \mathrm{s}$ (and $\mu$ is the same as suggested in $\$ 2.5 .2$ ), which yields a wall shear stress of $\tau_{\mathrm{w}}=5.3$ dynes $\mathrm{cm}^{-2}$; therefore no cell damage is expected for cells that tolerate the shear stresses in the circulatory system.

Molecular interactions can also be regulated by external forces. Flow-induced shear stress may provoke conformational changes in solved (or adsorbed) polymers [83], potentially leading to the exposure of otherwise buried molecular sequences (cryptic sites) [84], and/or to reduced or enhanced molecular interaction lifetimes (the latter interactions are called catch bonds) [85]. Consequently, a 
molecular surface coating that has been exposed to flow may promote cell spreading to a different extent than a coating that has not [84].

\subsubsection{Signaling assays: the challenge of compound addition to pre-attached cells}

Among all types of cell-based experiments carried out by the means of label-free biosensors, probably signaling assays demand the most deliberate sample handling strategy. One reason is that signaling assays inherently require high-throughput measurements and, therefore, a sample handling strategy which is compatible with high-throughput. Generally, the sensors are rendered cell-adhesive by creating a molecular coating on their surface via adsorption. Then cells are seeded in high density on the surface, and incubated for 24 hours at $37{ }^{\circ} \mathrm{C}, 5 \% \mathrm{CO}_{2}$ to obtain a confluent cellular monolayer. These steps can be carried out manually with a channel pipettor. Subsequently, a baseline is established with assay buffer above the cells and some compound is added to them to activate a signal transduction pathway. Importantly, receptor activation and subsequent signaling are detected through both horizontal and vertical dynamic mass redistribution (DMR) in the bottom region of the cells [13]. (Therefore, it is not required to provoke changes in the size of the cell-substrate contact area for detection, if vertical DMR is significant.) The challenge lies in how the compound is introduced above the cells, especially because DMR signals are rather small and may follow rapid kinetics $[79,86]$. Ideally, introduction should be highly reproducible, and the flow generated during introduction should not perturb the cells (compare with §2.4.2). Obviously, these criteria are hardly met if compounds are added manually with a pipettor; the introduction rate and the distance from cells are not well-defined and cannot be performed parallel in 96 or 384 channels. In closed cuvette systems (§4.1), therefore, primarily an integrated sample dispenser robot has been used for sample manipulation, which enables highly controlled and reproducible liquid exchange above cells (for a detailed protocol, see [79]). Cell signaling assays are considerably more straightforward on flowthrough fluidic systems [87]; here, cells should be plated on the pre-coated sensor surface, allowed to spread for a defined time interval, then a constant flow of buffer should be initialized and sustained 
throughout the assay. After baseline reading, any compound can be conveniently introduced into the flow. In addition, this system also enables to control the duration of the stimulation. However, highthroughput biosensors with a flow-through system have not hitherto been commercialized.

\section{Experimental: materials and methods}

\subsection{Optical waveguide lightmode spectroscopy}

Surface-sensitive optical biosensors take advantage of surface-bound electromagnetic waves to detect refractive index changes (evoked by either bulk refractive index changes, molecular adsorption, cell spreading, or dynamic mass redistributions in spread cells) close to a solid-liquid interface. Exactly how this is done is greatly dependent on the technique itself and the actual instrumental configuration. OWLS detection is based on a nano-grating planar optical waveguide (i.e. sensor chip, Fig. 3b), which is illuminated by a laser beam. The nano-grating embedded into the waveguide structure enables light to be incoupled into the waveguide layer, which then propagates several millimeters, permitting light intensity measurements at the ends of the sensor chip. Such waveguiding, however, can be achieved only at discrete illuminating angles, which are dependent on the refractive index of the sample layer being closest $(100-200 \mathrm{~nm})$ to the surface of the sensor chip. The illuminating angle is varied by rotating the waveguide with a high-precision goniometer relative

to the illuminating light beam (Fig. 1). Plotting the photointensity measured at the ends of the waveguide against the illuminating angle yield the OWLS spectrum; sharp resonant peaks with a typical width of $0.05-0.07^{\circ}$ indicate what angles waveguiding is achieved at (resonant angles). Whenever the refractive index over the sensor surface is altered, the position of the peaks in the spectra will be shifted. 
Throughout this study, experimental data are presented as the alteration of the effective refractive index of the zeroth order transverse magnetic lightmode (simply denoted as $\Delta N$ ). The effective refractive indices of the waveguide modes can be derived from the resonant angles $[16,88]$.

\subsection{Sensor chip preparation}

OW2400 OWLS sensor chips (Microvacuum Ltd., Hungary) were used in all experiments presented in this study. Sensor chips were cleaned according to the following protocol. Cellular contamination was first removed by sonicating the chips in an aqueous medium. The waveguides were then soaked in chromic acid for $3 \mathrm{~min}$, then rinsed with Milli-Q water (MQ), 0.5 M potassium hydroxide, and washed with copious amount of MQ water. The chips were placed into MQ water in sonicator for $30 \mathrm{~min}$ and the MQ was changed over them every $3 \mathrm{~min}$. Prior to experiments the waveguides were equilibrated in buffer overnight.

\subsection{Experiments on flow-through systems}

The prepared waveguides were mounted onto the measuring head of an OWLS instrument. Custom made polyether ether ketone (PEEK) cuvettes were then sealed to the waveguide with a Kalrez O-ring [89]. Flow was guided by tubes made of either silicone (Ismatec, Tygon R3607) or polytetrafluoroethylene (PTFE), with inner diameters of $0.51 \mathrm{~mm}$ or $0.8 \mathrm{~mm}$. The ends of Teflon tubes were flattened with an Omnifit kit (Biochem Fluidics) and connected via linear junctions. A porous hydrophobic membrane-based bubble trap (Omnifit) was integrated into the flow-through fluidic system.

When a large amount of sample was available (>2 ml), either a peristaltic pump (Reglo Digital, Ismatec) or a laboratory-built, computer-controlled syringe pump was used to generate continuous flow above the sensing area. In contrast, when the sample volume was limited, small amounts were injected into the fluidic setup either using an injection valve (i.e. SIS-06, see §4.3.1) or a septum injector (see §4.3.2). 
For practical reasons, glycerol (Spektrum 3D) solutions were used as sample in most OWLS experiments. Most importantly, the interaction of glycerol with the waveguide is completely and instantly reversible, i.e. the solution can be removed by flushing the fluidic system with MQ or PBS (phosphate-buffered saline, Sigma-Aldrich), resulting in restoration of the baseline. Hence, multiple experiments can be carried out consecutively with the same sensor chip. This is because glycerol only changes the bulk (cover) refractive index, i.e. it does not form an adlayer nor does it diffuse into the chip. For surface adsorption experiments poly-L-lysine (PLL, Sigma-Aldrich) and avidin (SigmaAldrich) solutions were used.

\subsection{Cell culture, cell adhesion studies}

3 T3 fibroblast cells were routinely cultured in Dulbecco's modified Eagle's medium (DMEM, Invitrogen) supplemented with $10 \%$ fetal bovine serum (FBS, Invitrogen), 4 mM L-glutamine, 0.25 $\mu \mathrm{g} / \mathrm{m}$ amphotericin, and $40 \mu \mathrm{g} / \mathrm{ml}$ gentamycin (culture medium). Cells were harvested using $1 \%$ trypsin and EDTA (Invitrogen). Trypsin activity was arrested with culture medium containing 10\% FBS, which was eventually replaced (centrifugation twice, $300 \mathrm{~g}, 5 \mathrm{~min}$ ) with serum-free medium buffered with $25 \mathrm{mM}$ 4-(2-hydroxyethyl)-1-piperazine-ethanesulfonic acid (HEPES, Invitrogen), $\mathrm{pH}$ 7.0. Cells were then seeded into a closed cuvette and their adhesion monitored for $4 \mathrm{~h}$. After the experiment, the cuvette was taken out from the OWLS measuring head, the medium above the spread cells was removed, the cuvette was disassembled, and the waveguide was positioned on a specially designed microscope insert. A cuvette having a bigger diameter than the OWLS cuvette can be mounted and fixed on this microscope insert, allowing replenishment of the medium above the cells. The whole area occupied by the cells was then scanned with the 10x objective of an Observer Zeiss microscope. 


\subsection{Fluidic simulations}

Computational fluid dynamics (CFD) computations were performed to obtain a detailed view of the flow field inside the cuvette. The ANSYS CFX commercial CFD package was used as a flow solver and ICEM CFD was employed for meshing. The mesh consisted of approximately $6 \times 10^{5}$ elements, which were mostly tetrahedral apart from the near-wall boundary layer cells, which were hexahedral. The momentum equations and the continuity equations were solved in a steady, incompressible formulation. The shear stress transport (SST) model was applied to cope with the turbulence, which blends between the k-epsilon and k-omega formulations. The volumetric flow rate was prescribed at the inlet (with a uniform inlet velocity profile) while an average static pressure was prescribed at the outlet, the rest of the surfaces in contact with the fluid being no-slip walls. High resolution (mostly second-order) spatial discretization was applied. The results were accepted once both the scaled average RMS error fell below $10^{-5}$ and the global imbalance of the conserved quantities reached $0.1 \%$.

\section{Modes of fluid introduction}

Fluid introduction is the action of filling the sample volume over the sensor with the solution of interest (e.g. flushing a flow-through cuvette, or pipetting medium into a closed cuvette). As it will be shown later, several types of errors in OWLS data and interpretation are associated with inappropriate introduction of the sample into the cuvette. How fluid introduction can best be carried out is mainly determined by the cuvette type and the amount of the available sample.

\subsection{Closed cuvette without flow and manual fluid introduction using a pipette: monitoring cell adhesion and spreading}

The simplest possible fluidic tool enabling the exposure of the sensing area to the solution of interest is the closed cuvette (Fig. 3). Samples have to be introduced manually using pipettes, and 
continuous flow cannot be generated in such an arrangement. However, all of the commercialized high-throughput optical biosensors employ open wells, and are not currently available with flowthrough systems $[14,68]$.

Typically, closed cuvettes are used when some activity (adhesion and spreading, proliferation, response to effector molecules, etc.) of cells are monitored [14,25,26,28,30]. Depending on the aim of the investigation, cellular assays on a biosensor may take up to hours or days, and the fewer disturbances to the system during the measurement the better. Contamination can easily be avoided by covering the cuvette with a piece of Parafilm, but several undesirable phenomena can still potentially perturb the system. Diffusion of gases into the cell suspension can cause $\mathrm{pH}$ changes and solvent evaporation may cause the osmolality of the medium to increase. The biosensor might directly respond to such changes, which furthermore stress the cells, changing their normal behavior (i.e. that observable in an optimal, well-defined and unchanging environment). Recently, an OWLS closed cuvette has been developed into a mini-incubator that enables the temperature and $\mathrm{pH}$ of the cell suspension to be automatically controlled [30]. This mini-incubator equipped OWLS system has been then used to monitor the spreading and adhesion of sensitive primary immune cells isolated from human blood [30].

The obvious caveat associated with the use of a closed cuvette system is the difficulty of performing manipulations on the sample. Nowadays one generally wishes to continue the work after the cells spread on the sensor surface, and monitor either their proliferation, survival or response to various effector molecules (drugs, ligands, toxins). It is known that not only the presence of the effector but also the duration of the stimulation is crucial in cell biology [90]. In contrast to the desirable fast, yet gentle and controllable sample exchange, cumbersome pipetting from a closed cuvette implies the relatively uncontrolled removal of only a portion of the bathing medium. In addition, when the cuvette is mounted on the rotating goniometer of an integrated optical scanner (incoupling configuration [16]) the scanning has to be stopped to perform any manipulation in the sample volume of the closed cuvette and, therefore, typically for tens of seconds following sample 
addition the response cannot be monitored. In an OWLS device without moving parts, such as the outcoupling configuration [17] or one of the various kinds of interferometry [7,17], the measurement would not have to be stopped, although there might be some optical perturbation due to fluid movements. Furthermore, washing steps cannot be conveniently carried out in the closed cuvette. To overcome the drawbacks of closed cuvettes, flow-through systems for living cell applications have been specifically designed $[77,90,87]$.

In summary, closed cuvettes are ideal for applications where sample manipulations during the measurement are barely needed - these applications include cell spreading assays, or drug screening assays that aim at demonstrating a drug effect on a cell population and the duration of stimulation is less important (Table 1). A typical experimental arrangement with a closed cuvette and a spreading curve obtained with OWLS are shown in Fig. 3. Here, fibroblast cells were seeded onto the sensor surface pre-coated with PLL, and their spreading was monitored for $4 \mathrm{~h}$.

\subsection{Fluidic systems with flow-through cuvettes}

Sophisticated fluidic setups can be built by connecting supplementary fluidic elements (bubble trap, junctions, pumps, etc.) to a flow-through cuvette (Fig. 1). Typically, the inlet tube connects the sample reservoir with the sample volume of the cuvette, and the outlet tube leads to a waste container

(Fig. 1, Fig. 4). Peristaltic or syringe pumps are used to generate flow ensuring a constant supply of material. Exchanging the samples is very simple and the flow rate can be easily set to the desired value - altogether the flow-through technique is particularly advantageous because of the experimental controllability and simplicity it offers [17,41]. Moreover, since the flow is continuous, OWLS data is easily recorded during both the adsorption and desorption phases of a molecular/cellular process under well-controlled conditions. Therefore, the kinetic rate coefficients of the processes can be easily determined by fitting kinetic models of more or less sophistication to the data. The role of flow from a biological point of view was considered in $\S 2.5 .3$; some investigations 
may have more biological significance if performed under flow, but this may substantially complicate data interpretation and/or experimental design.

Flow-through fluidics can, however, only be used when a sample amount sufficient for an entire experiment is available. With a typical flow rate in the range of microliters per second this usually means milliliters of solutions. Furthermore, given that OWLS (and DPI several orders of magnitude more so) is sensitive to changes in temperature and $\mathrm{pH}[47,89]$, it is critical to ensure that these parameters are the same for a subsequently introduced sample as those for the sample to be replaced. Moreover, some flow-through cuvettes might be inappropriate for working with mammalian cell suspensions due to geometrical issues; according to our experience, cells can adhere and aggregate in the immediate vicinity of the inlet aperture (before the sensing area), rather than being uniformly distributed on the entire bottom of the cuvette. Thus, careful scrutiny has to be given when designing a flow-through cuvette for cellular assays.

A flow-through system unavoidably hides risks in its relatively complicated arrangement and whole measurements can be endangered if the diverse constituent elements of the system are not carefully tested one by one and their incidental effects on the measurement revealed. Gas bubbles, for example, can grossly distort the data, but the integration of a bubble trap into the fluidic system offers an easy way to efficiently suppress this threat (\$4.2.2.3). Also, the inner diameter of tubes has to be chosen carefully: it is advantageous to use larger diameters closer to the cuvette, followed by smaller diameters at the pumps to effectively dampen possible abrupt pulsations/variations in flow.

The advantages, associated caveats and typical application areas of OWLS with flow-through fluidics are summarized in Table 1. 


\subsubsection{Basic building blocks of a flow-through setup}

\subsubsection{Cuvette and tubing materials}

As previously found [89], and now confirmed, silicone cuvettes leave contamination on the surface of the chip. We therefore used polyether ether ketone (PEEK) for the cuvettes and Kalrez (a perfluorinated elastomer) O-rings for sealings (Fig. 4), which are sufficiently inert to be recommended for all measurements.

Many materials, especially silicone, are unsuitable for the tubing. Those that are permeable may let gases diffuse into the sample, which may result in undesired bubble formation, especially when a peristaltic pump is used to generate flow (see §4.2.1.2). Tygon LF is soft enough to be suitable for peristalsis. PTFE (Teflon) is probably the best tubing material for connecting tubes, since it is resistant to most organic solvents and even to strongly acidic or basic solutions. However, PTFE is too rigid to be used with peristaltic pumping.

\subsubsection{Pumps}

Peristaltic and syringe pumps are commonly used for pressure pumping in biosensor experiments [91]. More sophisticated, more advanced experiments should be carried out with syringe pumps because they offer more controllability and more uniform flow. Our custom-built syringe pumps are driven by a program enabling fully automatic control over flow rate (including temporally varying ones), and the initiation, duration and termination of flow sequences. In addition, the entire flow system including the connecting tubes can be built from chemically resistant PTFE tubes (possibly in combination with syringes made of glass). However, the potential of syringe pumps can only be fully exploited if one has at least two of them (one for pumping the buffer and one for the analyte), because if the syringe in one pump has to be changed, the measurement would have to be stopped and the risk of introducing bubbles would arise. 
In contrast, a single peristaltic pump offers an easy method for generating quasi-uniform flow, but some commercially available peristaltic pumps (the ones with circular rather than elliptical roller races, or with fewer than $8-10$ rollers) tend to introduce pulsations in the flow that can influence both the sensor itself [92] and the adsorption or other process under investigation.

\subsubsection{Exchange of samples using flow-through fluidics}

Regardless of how carefully the samples are manipulated and exchanged, certain undesired effects cannot be eliminated and may significantly affect the recorded biosensor data, making their interpretation more difficult. Dead volumes are especially problematic $(\S 2.2)$. Here, we demonstrate how the diffusion processes taking place at various points of the fluidic system affect the measurement.

\subsubsection{Diffusion due to the dead volumes of the cuvette}

Most flow-through cuvettes have unflushable volumes between the sealing O-ring and the inlet apertures. With properly planned experiments it can be shown that these „dead volumes” have an important effect on the actual biosensor measurements (see Fig. 5) - especially when small amounts of sample are used - and consequently these volumes should be minimized. As a result of diffusion to and from the dead volumes, data collection just after changing the inflow from pure solvent to analyte solution may not represent the sample of interest.

Our test measurements were executed as follows. The baseline was established with either PBS or MQ, then pressure-driven flow of aqueous glycerol solution was initiated. The flow was suddenly stopped well before saturation of the OWLS signal, and a drastically decrease of the signal was observed (Fig. 5a). In the inverse experiment (Fig. 5b) the cuvette was initially fully filled with glycerol solution, which was then partially removed by pumping pure buffer for typically $0.5-1 \mathrm{~min}$. 
In this case stopping the buffer flow resulted in a rising biosensor signal, clearly indicating that glycerol was diffusing into the measuring zone from the unflushed volumes.

Here, we introduce a method to estimate the size of the dead volumes relative to the total cuvette volume. The effective refractive index $N$ is approximately linearly proportional to the change in refractive index of the cover layer $\left(n_{\mathrm{c}}\right)[88]$, i.e.

$$
\Delta N=\left(\frac{\partial N}{\partial n_{\mathrm{C}}}\right) \Delta n_{\mathrm{C}}
$$

Denoting the volume that is flushed with laminar flow (convective zone) at time $t$ by $V_{\mathrm{C}}(t)$ ), and the corresponding unflushed dead volume (diffusive zone) by $V_{\mathrm{D}}(t)$, the total volume of the cuvette is

$$
V_{\mathrm{T}}=V_{\mathrm{C}}(t)+V_{\mathrm{D}}(t)
$$

Let $\Delta N_{1}$ and $\Delta N_{2}$ be defined as in Fig 5a, and $\alpha$ be the constant of proportionality between the effective refractive index and the concentration of the sample. Using this notation, the amount of glycerol in the cuvette at the instant of stopping the flow is $V_{\mathrm{C}}(t) \alpha \Delta N_{2}$ and the total amount of glycerol following the equilibration of local concentration differences is $V_{\mathrm{T}}(t) \alpha \Delta N_{1}$. Building on the fact that the amount of glycerol present in $V_{\mathrm{T}}$ does not change after the flow is stopped, the two qauntities can be equated, yielding

$$
V_{\mathrm{D}}=\frac{V_{\mathrm{T}}\left(\Delta N_{2}-\Delta N_{1}\right)}{\Delta N_{2}} .
$$

Our experimental findings were qualitatively confirmed by computational simulations of the flow in one of our flow-through cuvettes (Fig. 4a). When the flow rate is low $(1 \mu \mathrm{l} / \mathrm{s})$, the flow is laminar and $V_{\mathrm{D}}$ is rather big (Fig. 6a). In contrast, if flow rates around $100 \mu 1 / \mathrm{s}$ are used, the flow becomes turbulent and most of the dead volumes are successfully eliminated (Fig. 6b, and see $\$ 4.3 .2)$. 


\subsubsection{Length of tubing}

The Hagen-Poiseuille-equation (Eq. 2) states that the flow resistance grows with tube elongation; one should, therefore, always check whether the original flow rate can be maintained if extra tubing is added. We used three tubes with lengths of 47, 147 and $447 \mathrm{~cm}$ and an inner diameter of $0.51 \mathrm{~mm}$. The actual flow rate was determined from the amount of sample collected at the end of the tubing and the collection time. Our pump was robust enough for flow rate not to diminish.

An earlier investigation found no effect of tube elongation on the sensor signal saturation time [93]; however, tubes with only slightly different lengths were used in that study $(17.3,22.3$ and 25.3 $\mathrm{cm})$. In contrast, we found that significantly more sample is necessary for reaching saturation of the OWLS signal (i.e., to completely fill the cuvette with the sample) when the inlet tube is longer, and suspected that this was an effect of diffusion. It is clear that the diffusion in the direction perpendicular to the axis of the tube is much more significant than diffusion parallel to it [94]: sample in the boundary layer along the walls of the tube is exchanged by diffusion instead of convection.

The time the fluid spends in the tubing (i.e., the average time available for diffusion) is proportional to the tubing length $L(t \sim L)$. The distance the sample diffuses perpendicular to the diffusion boundary layer is proportional to the square root of the time spent in the tubing (Eq. 3), hence saturation time of the signal $\left(t_{\mathrm{s}}\right)$ is expected to increase proportionally to the diffusion time $t$. Saturation times measured at different tubing lengths and plotted against the square root of tubing lengths can be nicely fitted with a straight line $\left(t_{\mathrm{s}} \sim L^{2}\right)$; thus the experimental data nicely supports the above prediction (Fig. 7).

\subsubsection{Eliminating bubbles - effect of a bubble trap}


A bubble forming inside the fluidic channel has a grossly different refractive index compared to the liquid medium or analyte and its presence will, therefore, severely distort the biosensor experiment - therefore great care has to be taken to avoid bubbles. Sonication, filtration and vacuum treatment of the solutions degas them and, hence, reduce the probability of bubble formation. Wider tubing followed by a smaller one helps to prevent bubbles forming at the junctions between tubes.

Another possibility is to incorporate a bubble trap into the fluidic setup [47]. Although the inner part of the bubble trap contains multiple arcs in which the sample is guided, we found that it has no undesirable mixing properties. Only a slight increase in $\tau$ was observed when the bubble trap was integrated into each of the three tubes having different lengths $(47,147$ and $447 \mathrm{~cm})$ as compared to the cases when we did not use a bubble trap. The increase corresponded to an increased tubing length: a $15 \mathrm{~cm}$ long extra section of tubing was inserted to integrate the bubble trap, which itself contributes the equivalent of an additional $15 \mathrm{~cm}$ (the approximate length of its arcs). This is evidenced in Fig. 7, as these additional data points, marked with "bt", are well fitted by the model described in $\$ 4.2 .2 .1$.

\subsection{Injection systems for the introduction of limited amount of sample}

Some samples are scarce or highly expensive and, therefore, only very limited amounts may be available. The minimum amount of sample necessary for an experiment (which normally means enough to obtain the kinetics up to steady state) can be effectively decreased if the sample is not pumped through the whole fluidic setup but injected closer to the sensor. It should be stressed, however, that small sample amounts are more prone to attenuation caused by diffusion $(\S 4.2 .2 .2)$.

A combination of the reproducible and precise fluid handling characteristic of flow injection analysis (FIA) with the sensitive optical detection offered by OWLS resulted in the development of FIA-OWLS immunoassays. An injection valve (§4.3.2) and manual injections have been used to introduce samples into the cuvette [95]. For the development of OWLS immunosensors an FIA 
system with a peristaltic pump, an injector valve and an injector loop have been employed [96,97]. Various lab-built flow-through cuvettes and injection ports have also been tested to reproducibly introduce low amount of samples. In polyelectrolyte studies normally $100 \mu \mathrm{L}$ sample solution was manually introduced to flush a $37 \mu \mathrm{L}$ cuvette $[98,99,100]$. Elsewhere, a sequence of adsorption steps has been used to maximize adsorption from a given sample quantity [101,102,103]. Very recently, the sophisticated FastStep ${ }^{\mathrm{TM}}[104]$ and OneStep $^{\text {TM }}[105]$ injection systems have been developed. In the former configuration the sample and buffer streams merge right before the flow cell, and a stepped analyte concentration profile is produced by varying the flow rate ratio of the two branches. In the latter configuration, the undiluted analyte disperses in a capillary tube before entering the cuvette, thereby producing a concentration gradient. In contrast to standard fixed concentration injections, where a set of dilutions is required to complete a dose response range, both of these systems generate a full dose response from a single analyte injection, thereby reducing the variability, hence systematic error, among experiments. Therefore, these systems enable a gradient in $\mathrm{pH}$, salt, or co-factors for rapid optimization of buffer conditions to be conveniently titrated.

In this paper the SIS-06 injection valve supplied to a BIO-210 OWLS instrument and a septum injector are tested and discussed in more detail.

\subsubsection{Injection valves}

The SIS-06 injection valve (Fig. 8) from MicroVacuum Ltd. can optionally be integrated between a pump and a regular flow-through cuvette to reduce the amount of sample necessary for a measurement. Both the buffer and the limited amount of sample are transferred into the cuvette by continuous pump-driven flow. The injector has 6 channels, and the exact route of flow depends on the operation mode set. The injection valve operates in two modes. In the "Load Position" the sample loop can be filled with the analyte, while the buffer is conveniently flowed towards the cuvette through two channels which bypass the sample loop. In the "Injection Mode" the sample loop is 
connected to the pressure-driven buffer flow and its content is transferred to the cuvette. Calibrated sample loops are commercially available with different volumes. Throughout this study we used one with a volume of $50 \mu 1$.

First we injected 5.8\% glycerol into the fluidic setup via the SIS-06 injector valve (Fig. 9a). The glycerol sample $(n=1.33518)$ and MQ water $(n=1.33085)$ was pumped over the sensor with a programmable syringe pump at different flow rates $(1.44,0.7$ and $0.14 \mu \mathrm{l} / \mathrm{s})$. Despite the fact that the volume of the cuvette was only approximately $20 \mu \mathrm{l}$, the injected $50 \mu$ of sample seemed to be insufficient to flush the cuvette through, we could not saturate the signal using any of these flow rates (Fig. 9a). We suspected that this was a consequence of the presence of unflushable volumes in the tubes and the cuvette $(\S 4 \cdot 2 \cdot 2.1)$.

The minimal sample amounts necessary to saturate the signal at each flow rate were determined in separate experiments, in which an unlimited amount of sample was pumped until a signal plateau was obtained (Fig. 9b). Surprisingly, more than $200 \mu$ l sample was required when a flow rate of 1.4 $\mu 1 / \mathrm{s}$ was used, and only slightly less was needed if the flow rate was $0.14 \mu 1 / \mathrm{s}$. This underlines the importance of optimizing of the fluidic arrangements before injecting small amount of samples, in order to obtain relevant results without wasting material.

In order to minimize diffusional effects and waste, the fluidic setup has been rearranged according to the conditions discussed in $\S 4$ : we have i) changed tubes to ones having smaller inner diameters (from $0.8 \mathrm{~mm}$ to $0.51 \mathrm{~mm}$ ); ii) designed a cuvette having a smaller sample volume (approximately $15 \mu 1$ ); iii) minimized the tubing length between the valve and cuvette to $4.5 \mathrm{~cm}$; iv) chosen low flow rates, and v) integrated a bubble trap in the system. We then performed a new experiment with the biosensor and used the injection valve for sample introduction. Using this optimized fluidic system we were finally able to successfully measure the presence of $50 \mu 1$ of $6 \%$ glycerol (Fig. 10), but only when the flow rate was at most $0.14 \mu 1 / \mathrm{s}$ (as discussed earlier, a smaller amount of sample is enough to saturate the signal when a lower flow rate is used). 
It should be realized, however, that the SIS-06 injector used with continuous flow is less suitable for adsorption or affinity-binding studies, because these processes generally take more time than for which the sample could be possibly present in the cuvette. Keeping the sample longer in the cuvette by lowering the flow rate is strongly discouraged, because the binding process would be transport limited. Stopping the flow when the cuvette is completely filled with the analyte solution may be a better option, but careful calibration is needed to determine the exact time the flow has to be stopped (because protein solutions often do not alter the signal immediately).

\subsubsection{Septum injector}

A special cuvette called septum injector (Fig. 4/d,e) enable limited amounts of sample to be directly injected over the sensor. To introduce the sample, a membrane on one of the stalks of the cuvette has to be pierced with a special septum needle; the withdrawal of the needle allows the membrane to self-seal.

With measurements using $50 \mu \mathrm{l}$ of $6 \%$ glycerol (Fig. 11a) we demonstrated how the problem of dead volumes could be eliminated by a fast injection rate, which was sufficient to effectively flush the dead volumes (Fig. 6). The adsorption of $50 \mu 1$ of $50 \mu \mathrm{g} / \mathrm{ml}$ avidin rapidly introduced via the septum injector also gave satisfactory results (Fig. 11b). Desorption required multiple injections of pure buffer, which, however, generates complicated kinetics. Hence, it is desirable to flow buffer continuously (which is usually available in unlimited quantity) to obtain a monotonic desorption signal, which then can be analyzed to determine the kinetic parameters [106]. To achieve this, we removed the needle of a septum syringe and introduced it into a piece of tubing (Fig. 4/e), which could be readily used to connect the pump and the septum cuvette, thus enabling continuous washing (Fig. 12). This modification eliminates transport-limited retardation of the initial adsorption rate. 


\section{Conclusions}

One of the most important advantages of label-free biosensors is that they generate kinetic data, thus allow for kinetic data analysis. This, in turn, can contribute to a better understanding of the mechanisms underlying the investigated surface process (may it be cell spreading, molecular adsorption, receptor-ligand interactions, etc.) - which is ideally the final aim of every study in the field. It should be realized, however, that the way the liquid samples are handled (introduced, exchanged) may deeply influence both the kinetics of the surface process, and the detection limit of the biosensor. The fluidic system is the most critical part of any biosensor setup, since the majority of experimental artefacts and misinterpretations of the data generally originate in an inappropriate fluid handling strategy or in the negliance of fluidic effects that severely interfere with the kinetics of the true surface process.

In an adsorption or receptor-ligand binding assay, for instance, the obtained biosensor signal will not be dominated by the kinetics of the true surface process, unless transport limitation of the analyte $(\S 2.1, \S 2.3 .1)$ and the flushing effect $(\S 2.3 .1)$ are both successfully eliminated. Diffusion to the diffusion boundary layers $(\S 4.2 .2 .2)$, or to the dead volumes $(\S 4.2 .2 .1, \S 4.3 .1)$ of the cuvette may cause the analyte solution to significantly attenuate, therefore the sample concentration in the sensing zone will be ill-defined, precluding the correct interpretation of the obtained kinetic data. (When adsorbing macromolecule solutions are measured, the effect of attenuation may be masked by adsorption; data analysis ignoring this distortion is likely to lead to serious error.) These phenomena, illustrated with experimental data and discussed in $\S 4.2$, have an increasingly decisive role when working with strongly limited amount of samples $(\S 4.3 .1)$. These effects can be most successfully reduced by generating turbulent flow in a septum injector-based fluidic system $(\S 4.3 .2$, Fig. 6); this efficiently eliminates those volumes which cannot be flushed with laminar flow. If necessary, the dead volumes can be further decreased if a bigger amount of solution is injected into the cuvette. However, the efforts to minimize the necessary amount of sample for the actual measurement and to flush the cuvette perfectly are, to some degree, inconsistent with each other; consequently, one 
always has to make a compromise. If the sample of interest is very expensive and/or scarce, calibration with a model solution (e.g. glycerol), and subsequent correction of the signal of the actual sample can be the key for more accurate measurements. In the future, further miniaturization of the instrumentation may take place and, when molecular samples are being investigated, the tube dimensions may be further reduced down to the nanoscale. This introduces certain peculiarities that should then be explicitly considered $[107,108]$.

Sample handling of cell suspensions may be even more challenging than that of analyte solutions ( $\$ 2.4)$, especially because cells are capable to sensitively respond to the changes of their surroundings and actively modify their environment. Ideally, both the temperature and $\mathrm{pH}$ of the bathing medium should be kept at constant levels, preferably close to that experienced by cells in vivo; this requires further developments on a basic fluidic system. Interpretation of kinetic cellular data may be complicated because optical biosensors detect refractive index changes in a non-specific manner (§3.1). Considering a cell spreading assay, the biosensor signal provoked by cell spreading may be superposed by an adsorption signal if cellular secretion adsorb or bind to the sensor surface or the molecular coating (§2.4.1). Monitoring other cellular activities may demand even more considerations $(\S 2.4 .2, \S 2.4 .3)$. Appropriate flow-through fluidic systems enable cellular mechanotransduction to be monitored with a biosensor (Fig. 1), but different cell types may require different experimental design. Those cells which are constantly exposed to the shear stresses in the circulatory system are not expected to suffer cell damage in a typical biosensor experiment, but this needs careful scrutiny for other cell types $(\S 2.5 .2)$.

In summary, when measurements are carried out by means of biosensors, it is critical to establish a reliable strategy and a well-tested fluidic (§4) system for controllable and reproducible fluid handling. There seems to be no ideal fluidic design, which is optimal for every application; each should be fitted to one another. In $\S 4$ we reviewed the most common fluidic systems and components used in biochemical surface sensing, illustrated their performance with experimental data, and discussed their advantages and disadvantages; our final conclusions are summarized in Table 1. 


\section{Acknowledgements}

The support of the Hungarian Scientific Research Fund (OTKA-PD 73084) and the European Commission (ERG OPTIBIO) is gratefully acknowledged. Part of this work was carried out in the framework of an European Commission-supported project, aiming blood analysis using optical biosensors (P3SENS). This work was supported by the Lendület program of the Hungarian Academy of Sciences, and the Bolyai Scholarship to B. S. from the Hungarian Academy of Sciences.

This research was realized in the frames of TÁMOP 4.2.4. A/2-11-1-2012-0001 „National Excellence Program - Elaborating and operating an inland student and researcher personal support system convergence program. The project was subsidized by the European Union and co-financed by the European Social Fund.

\section{References}

[1] Marks RS, Cullen DC, Karube I, Lowe CR, Weetall HH (editors). Handbook of Biosensors and Biochips. Wiley; 2007.

[2] Constable EC, Harverson P, Ramsden JJ. Chem Commun 1997;-:1585-1692.

[3] Steiner G. Anal Bioanal Chem 2004;379:328-331. 
[4] Scarano S, Mascini M, Turner AP, Minunni M. Biosens Bioelectron 2010;25:957-966.

[5] Homola Y, Yi SS, Gauglitz G. Sensor Actuat B - Chem, 1999;54:3-15.

[6] Marx KA. Biomacromolecules, 2003;4:1099-1120.

[7] Freeman NJ. Dual-polarization interferometry: An optical technique to measure the orientation and strucutre of proteins at the solid-liquid interface. In: Dejardin Ph, editor. Proteins at Solid-Liquid Interfaces, Heidelberg: Springer-Verlag; 2006; p.75-104.

[8] Ramsden JJ. High resolution molecular microscopy. In: Dejardin Ph, editor. Proteins at SolidLiquid Interfaces, Heidelberg: Springer-Verlag; 2006; p. 23-49.

[9] Kozma P, Hámori A, Kurunczi S, Cottier K, Horvath R. Sensor Actuat B-Chem 2011;155:446450.

[10] Patko D, Cottier K, Hamori A, Horvath R. Opt Express 2012;20:23162-23173.

[11] Patko D, Gyorgy B, Nemeth A, Szabó-Taylor KE, Kitteld A, Buzas EI, Horvath R. Sensor Actuat B-Chem 2013;188:697-701.

[12] Patko D, Mártonfalvi Zs, Kovacs B, Vonderviszt F, Kellermayer M, Horvath R. Actuat B-Chem 2014 accepted manuscript. 
[13] Fang Y, Ferrie AM, Fontaine NH, Mauro J, Balakrishnan J. Biophys J 2006; 91:1925.

[14] Orgovan N, Peter B, Bősze Sz, Ramsden JJ, Szabó B, Horvath R. Scientific Reports 2014;4:4034. DOI: $10.1038 /$ srep04034

[15] Orgovan N, Kovacs B, Farkas E, Szabó B, Zaytseva N, Fang Y, Horvath R. Appl Phys Lett 2014;104:083506. DOI: 10.1063/1.4866460

[16] Tiefenthaler K. Advanced Biosensors 1992;2:261-289.

[17] Ramsden JJ. J Stat Phys 1993;73:853-877.

[18] Vörös J, Ramsden JJ, Csúcs G, Szendrő I, De Paul SM, Textor M, Spencer ND. Biomaterials 2002;23:3699-3710.

[19] Ball V, Lustig A, Ramsden JJ. Phys Chem Chem Phys (PCCP) 1999;1:3667-3671.

[20] Ramsden JJ. Colloids Surf A 2000;173:237-249.

[21] Guemouri L, Ogier J, Zekhnini Z, Ramsden JJ. J Chem Phys 2000;113:8183-8186.

[22] Kovács N, Patko D, Orgovan N, Kurunczi S, Ramsden JJ, Vonderviszt F, Horvath R. Anal Chem 2013;85:5382-5389.

[23] Horvath R, Pedersen HC, Skivesen N, Selmeczi D, Larsen NB. Opt Lett 2003;28:1233-1235. 
[24] Yeh YP, Ramsden JJ. J Biol Phys Chem 2010;10:53-54.

[25] Ramsden JJ, Li S-Y, Heinzle E, Prenosil JE. Biotechnol Bioeng 1994;43:939-945.

[26] Li S-Y, Ramsden JJ, Prenosil JE, Heinzle E. Biotech Progr 1994;10:520-524.

[27] Ramsden JJ, Li S-Y, Heinzle E, Prenosil JE. Cytometry 1995;19:97-102.

[28] Horvath R, Pedersen HC, Skivesen N, Selmeczi D, Larsen NB. Appl Phys Lett 2005;86.

[29] Aref A, Horvath R, McColl J, Ramsden JJ. J Biomed Opt 2010;14:010501.

[30] Orgovan N, Salanki R, Sandor N, Bajtay Zs, Erdei A, Szabó B, Horvath R. Biosens Bioelectron 2014;54:339-344.

[31] Ramsden JJ. Phil Mag B 1999;79:381-386.

[32] Horvath R, Fricsovszky G, Papp E. Biosens Bioelectron 2003;18:415-428.

[33] Ramsden JJ, Lvov YA, Decher G. Thin Solid Films, 1995;254:246-251.

[34] Brynda E, Houska M, Skvor J, Ramsden JJ. Biosens Bioelectron 1998;13:165-172.

[35] Horvath R, Pedersen HC, Cuisinier FJG. Appl Phys Lett 2006;88:111102. 
[36] Ramsden JJ, Mate M. J Chem Soc Faraday Trans 1998;94:783-788.

[37] Mate M, Ramsden JJ. J Chem Soc Faraday Trans 1998;94:2813-2816.

[38] Ramsden JJ, Wright CS. Glycoconj J 1995;12:113-121.

[39] Kurunczi S, Hainard A, Juhasz K, Patko D, Orgovan N, Turck N, Sanchez JC, Horvath R. Sensor Actuat B-Chem 2013;181:71-76.

[40] Ramsden JJ. Kinetics of protein adsorption. In: Malmsten M, editor. Biopolymers at Interfaces, Ch. 10. New York: Dekker; 1998. p 321-361.

[41] Ramsden JJ, Bachmanova GI, Archakov AI. Phys Rev E 1994;50:5072-5076.

[42] Döbereiner H-G, Dubin-Thaler B, Giannone G, Xenias HS, Sheetz MP. Phys Rev Lett 2004;93:108105.

[43] Döbereiner H-G, Dubin-Thaler BJ, G. Giannone, Sheetz MP. J Appl Phys 2005;98: 1542-1546.

[44] Dubin-Thaler BJ, Hofman JM, Cai Y, Xenias H, Spiealman I, Shneidman AV, David LA, Döbereiner H-G, Wiggins CH, Sheetz. MP. PloS One 2003;3:1-15.

[45] Cuvelier D, Théry M, Chu Y-S, Dufour S, Thiéry J-P, Bornens M, Nassoy P, Mahadevan L. Curr Biol 2007;17:694-699.

[46] Aref A, Horvath R, Ramsden JJ. J Biol Phys Chem 2010;10:1-7. 
[47] Saini S, Kurrat R, Prenosil JE, Ramsden JJ. J Phys D: Appl Phys 1994;27:1134-1138.

[48] Valcarcel M, Luque de Castro MD. Fresenius J Anal Chem 1990;337:662-666.

[49] Oroszlan P, Thommen C, Wehrli M, Duveneck G, Ehrat M. Anal Meth Instr 1993;1:43-51

[50] Afeyan NB, Gordon NF, Regnier FE. Nature 1992;358:603-604.

[51] Levich VG. Physicochemical Hydrodynamics. Englewood Cliffs; 1962. NJ: Prentice Hall.

[52] Németh A, Kozma P, Hülber T, Kurunczi S, Horvath R, Petrik P, Muskotál A, Vonderviszt F, Hős Cs, Fried M, Gyulai J, Bársony I. Sensor Lett 2010;8:730-735.

[53] Brody JP, Jager P, Goldstein RE, Austin RH. Biophys J 1996;71:2338-2345.

[54] Schuck P. Biophys J 1996;70:1230-1249.

[55] Sjoelander S, Urbaniczky Cs. Anal Chem 1991;63:2338-2345.

[56] Davies PF. Physiol Rev 1995;75:519-560.

[57] Ingham DB. Aerosol Sci 1975;6:125-132.

[58] Schaaf P, Voegel J-C, Senger B. Ann Phys 1998;23:1-89. 
[59] Handbook of surface plasmon resonance, $2^{\text {nd }}$ edition. Editors: Schasfoort RBM, Tudos AJ. RSC Publishing 2008;chapter V: Kinetic and thermodynamic analysis of ligand-receptor interactions: SPR applications in drug development

[60] Biopolymers at interfaces, $2^{\text {nd }}$ edition. Editor Malmsten M. 2003:chapter VIII: Protein adsorption kinetics

[61] Nieba L, Krebber A, Plückthun A. Anal Biochem 1996:234;155-165

[62] Schuck P, Minton AP. Anal Biochem 1996:240;262-272.

[63] Curtis AS, Forrester JV. J Cell Sci 1984;71:17-35.

[64] Horbett TA. Principles underlying the role of adsorbed plasma proteins in blood interactions with foreign materials. Cardiovasc Path, ch. 1993;13(2):137S-148S

[65] Kurrat R, Walivaara B, Marti A, Textor M, Tengvall P, Ramsden JJ, Spencer ND. Colloid Surface B 1998;11:187-201.

[66] Wilson CJ, Richard BE, Clegg RE, Leavesley DI, Pearcy MJ. Tissue Eng 2005;11:1-18.

[67] Ramsden JJ, Horvath R. J Rec Sign Trans Res 2009;29:211-223.

[68] Fang Y. Assay and Drug Development Technologies 2006;4:583-595. 
[69] Frantz C, Stewart KM, Weaver VM. J Cell Sci 2010;123:4195-200.

[70] Hynes RO. Cell 1992;69:11-25.

[71] VandeVondele S, Vörös J, Hubbell JA. Biotechnol Bioeng 2003;82:784-790.

[72] Cottier K, Horvath R. Appl Phys B 2008;91:319-327.

[73] Horvath R, Cottier K, Pedersen HC, Ramsden JJ. Biosens Bioelectron 2008;24:805-810.

[74] Senger B, Bafaluy FJ, Schaaf P, Schmitt A, Voegel J-C. P Natl Acad Sci USA 1992;89:94499453.

[75] Choi HS, Talbot J, Tarjus G, Viot P. J. Chem. Phys. 1993;99:9296-9303.

[76] Vunjak-Novakovic RG, Obradovic B, Martin I, Bursac PM, Langer R, Freed LE. Biotechnol Prog 1998;14:193-202.

[77] Hug TS, Prenosil JE, Morbidelli M. Biosens Bioelectron, 2001;16:865-874.

[78] Frisch T, Thoumine, O. J Biomech 2002;35:1137-1141. 
[79] Schröder R, Schmidt J, Blättermann S, Peters L , Janssen N, Grundmann M, Seemann W, Kaufel D, Merten N, Drewke C, Gomeza J, Milligan G, Mohr K, Kostenis E. Nature Prot 2011;6:1748-1760. DOI:10.1038/nprot.2011.386

[80] Ingber DE. The FASEB J 2006;20:811-827.

[81] Papoutsakis ET. Tibtech 1991;9:427-437.

[82] Wong AD, Ye M, Levy AF, Rothstein JD, Bergles DE, Searson PC. Front neuroeng DOI: 10.3389/fneng.2013.00007.

[83] Ashton L, Dusting J, Imomoh E, Balabani S, Blanch EW. Biophys J 2010;98:707-714.

[84] Zhu C, Lou J, McEver RP. Biorheology 2005;42:443-462.

[85] Forero M, Thomas WE, Bland C, Nilsson LM, Sokurenko EV, Vogel V. Nano Lett 2004;4:1593-1597.

[86] Fang Y, Li G, Ferrire AM. J Pharmacol Toxicol 2007:55;314-322

[87] Goral V, Wu Q, Sun H, Fang Y. FEBS Lett 2011;585:1054-1060.

[88] Tiefenthaler K, Lukosz W. J Opt Soc Am B 1989;6:209-220. 
[89] Kurrat R, Textor M, Ramsden JJ, Boni P, Spencer ND. Rev Sci Instrum 1997;68:2172-2176.

[90] Zaytseva N, Miller W, Goral V, Hepburn J, Fang Y. Appl Phys Lett 2011;98.

[91] Osuga T. Jap J Appl Phys 1999;38:6564-6567.

[92] Csúcs G. The application of OWLS to Biomembrane-Protein Interactions; PhD dissertation ETH Zürich 1998.

[93] Brusatori MA. Protein adsorption kineitcs under an applied electric field: an optical waveguide lightmode spectroscopy study; PhD dissertation Wayne State University, Detroit, Michigan 2001.

[94] Taylor G. Proc R Soc A 1953;219:186-203.

[95] Trummer N, Adányi N, Váradi M, Szendrö I. J Anal Chem 2001;371:21-24.

[96] Székács A, Trummer N, Adányi N, Váradi M, Szendrő I. Anal Chim Acta 2003;487:31-42.

[97] Adányi N, Váradi M, Namsoo K, Szendro I. Curr Appl Phys 2006;6:279-286.

[98] Picart C, Ladam G, Senger B, Voegel J-C, Schaaf P, Cuisinier FJG, Gergely C. J Chem Phys 2001;115:1086-1094.

[99] Lavalle Ph, Gergely C, Cuisinier FJG, Decher G, Schaaf P, Voegel J-C, Picart C. Macromol 2002;35:4458-4465. 
[100] Boura C, Menu P, Payan E, Picart C, Voegel J-C, Muller S, Stolt JF. Biomaterials $2003 ; 24: 3521-3530$.

[101] Ramsden JJ, Dreier J. Biochem 1996;35:3746-3753.

[102] Van Tassel PR, Viot P, Tarjus G, Ramsden JJ, Talbot J. J Chem Phys 2000;112:1483-1488.

[103] Lionello A, Josserand J, Jensen H, Girault HH. Lab on a Chip 2005;5:1096-1103.

[104] Rich RL, Quinn JG, Morton T, Stepp JD, Myszka DG. Anal Biochem 2010;407:207-277.

[105] Quinn JG. Anal Biochem 2012;421:401-410.

[106] Schuck P, Minton AP. Anal Biochem 1996;240:262-272.

[107] Schock RB, Tongyoon H, Renand P. Rev Mod Phys 2008;80:839-883.

[108] Gogsadze R, Prangishvili A, Kervalishvili P, Chikovani R, Gogichashvili V, Jibladze N. Nanotech Perceptions 2013;9:57-69. 


\section{Tables}




\begin{tabular}{|c|c|c|c|c|c|}
\hline & \multicolumn{5}{|c|}{ cuvette types } \\
\hline & \multirow{3}{*}{$\begin{array}{l}\text { closed cuvette } \\
\quad \text { (Fig.3) }\end{array}$} & \multicolumn{4}{|c|}{ flow-through cuvettes (Fig 1, Fig. 4/a,b,c,e,f) } \\
\hline & & \multicolumn{2}{|c|}{ pump systems } & \multicolumn{2}{|c|}{ injector systems } \\
\hline & & peristaltic pump & syringe pump & SIS-06 (Fig. 8) & septum (Fig.4/d,e ) \\
\hline \multirow[b]{2}{*}{ advantages } & \multirow{2}{*}{$\begin{array}{l}\text { - temperature } \\
\text { control } \\
\text { - very simple, } \\
\text { easy-to-use tool } \\
\text { - ideal for } \\
\text { adhesion and } \\
\text { spreading studies }\end{array}$} & \multicolumn{2}{|c|}{$\begin{array}{l}\text { - continuous washing } \\
\text { - additional fluidic elements can be integrated } \\
\text { - may have more biological significance }\end{array}$} & \multicolumn{2}{|c|}{$\begin{array}{l}\text { - measurements can be carried out with small amount of } \\
\text { sample (i.e. with highly expensive, scarce samples) }\end{array}$} \\
\hline & & $\begin{array}{l}\text { - easy-to-use, user friendly } \\
\text { tool }\end{array}$ & $\begin{array}{l}\text { - fully automated } \\
\text { measurements } \\
\text { - whole tubing can be made } \\
\text { of PTFE }\end{array}$ & $\begin{array}{l}\text { - sample loops with different } \\
\text { calibrated volumes } \\
\text { - two operation modes (direct } \\
\text { injection/continuous flow) } \\
\text { - easy sample manipulation }\end{array}$ & $\begin{array}{l}\text { - the necessary amount of } \\
\text { sample is minimized } \\
\text { - unites all advantages of } \\
\text { every discussed tool } \\
\text { - relatively cheap }\end{array}$ \\
\hline \multirow{3}{*}{$\begin{array}{l}\text { dis- } \\
\text { advantages }\end{array}$} & \multirow{3}{*}{$\begin{array}{l}\text { - risk of } \\
\text { contamination } \\
\text { - sample } \\
\text { evaporation } \\
\text { - gas dissolvation } \\
\text { - manipulations in } \\
\text { sample volume } \\
\text { are cumbersome } \\
\text { - allows only } \\
\text { static stimulation }\end{array}$} & \multicolumn{4}{|c|}{$\begin{array}{l}\text { - dead volumes act like reservoirs when samples are changed } \\
\text { - complexity: all elements have to be tested carefully one by one } \\
\text { - air bubbles can remain/appear between junctions in the system } \\
\text { - temperatures and pH have to be the same for all subsequently introduced samples }\end{array}$} \\
\hline & & \multicolumn{2}{|c|}{$\begin{array}{l}\text { - significant amount of sample is necessary } \\
\text { - cell suspension cannot be reproducibly introduced } \\
\text { - long tube lengths: diffusion has a significant effect when } \\
\text { samples are changed }\end{array}$} & \multicolumn{2}{|c|}{$\begin{array}{l}\text { - efforts to minimalize the necessary amount of sample and to } \\
\text { flush the cuvette perfectly are inconsistent with each other }\end{array}$} \\
\hline & & $\begin{array}{l}\text { - pulsation in the flow if the } \\
\text { number of pump rollers is } \\
\text { less than } 10 \\
\text { - PTFE tubing cannot be } \\
\text { used for peristalsis }\end{array}$ & $\begin{array}{l}\text { - at least two syringe pumps } \\
\text { are needed to fully exploit } \\
\text { their potential }\end{array}$ & $\begin{array}{l}\text { - original arrangement is } \\
\text { unsuitable for protein } \\
\text { adsorption studies }\end{array}$ & - limited lifetime \\
\hline $\begin{array}{l}\text { typical } \\
\text { applications }\end{array}$ & $\begin{array}{c}\text { monitoring cell } \\
\text { adhesion and } \\
\text { spreading }\end{array}$ & \multicolumn{4}{|c|}{$\begin{array}{l}\text { All kinds of biosensor experiments can be performed and monitored: protein adsorption, ligand-receptor } \\
\text { binding, protein - lipid bilayer interactions, protein-DNA interactions, bio-compatibility study, cell response studies }\end{array}$} \\
\hline
\end{tabular}




\section{Figure captions}

Figure 1. Schematics (not to scale) showing an OWLS device with a basic flow-through fluidic system (bottom); and the bottom half of the parabolic flow profile inside the cuvette (top). Any kind of surface process is investigated, the reliability of the kinetic data is severely dependent on the appropriate fluidic design and on a considerate and reproducible fluid handling strategy.

At the bottom: the inlet tube connects the OWLS flow-through cuvette (mounted on the goniometer) with a sample reservoir. In between, a bubble trap is seen. The constant flow of analyte is engendered with a peristaltic pump, and the outlet tube leads the overflow to a waste container. Although this is considered to be a basic fluidic system, its constituting elements, or the way the liquid samples are handled may severely influence the kinetics of the surface process which has to be quantified.

At the top: analyte transport and shear stresses in the cuvette. In biosensorics, the engendered flow is typically laminar and, thus, the flow profile is parabolic. Close to the walls of the tubing or that of the cuvette, convection is negligible and solutions are exchanged via diffusion; this layer is called diffusion boundary layer (arrow to the left). An analyte (e.g. a protein) may adsorb reversibly or irreversibly to the sensor surface, or may desorb from there (with corresponding rate coefficients of $k_{\mathrm{r}}, k_{\mathrm{i}}$ and $k_{\mathrm{d}}$ ); causing changes in the local refractive index, which is eventually detected. The biosensor also enables the presence and activity of living cells seeded on the sensor surface to be monitored, although generally only the bottom portion of the cell can be probed (i.e. the sensing depth - shown as a reddish layer, and marked with a red arrow to the right - is only 100-200 nm). OWLS in combination with appropriately designed flow-through fluidic systems can be used to apply well-defined shear forces $\left(F_{s}\right)$ onto cells attached to the sensor surface, and monitor their response (cellular mechanotransduction). 
Figure 2. Illustration of the discrepancy between the adsorption kinetics predicted by a simple model (E q.5) (broken curve) and the kinetics most often observed in experiments (solid curve). The initial stage of the process is transport limited if the flow cannot effectively replenish the adsorbing particles, or the diffusion is slow compared to the width of the diffusion boundary layer.

Figure 3. Closed cuvettes in biosensorics. a) Image of a closed cuvette (shown both in assembled and disassembled form): i) cuvette- and metal chip holder, ii) waveguide (highlighted in red), iii) side wall of the cuvette, iv) retaining screw.

b) Closed cuvette used in OWLS experiments. i-iii) Schematic representation of the functional parts of an OWLS waveguiding sensor chip: supporting substrate, waveguiding film, grating incoupler, respectively. iv) Closed cuvette sealed by an O-ring to the surface of the waveguide forms the sample volume. v) Culture medium with which the OWLS baseline is established before monitoring of cell spreading. The cell suspension is introduced into the closed cuvette manually using a pipette. vi) OWLS signal obtained by monitoring cell spreading. The waveguide was coated with PLL (150 $\mu \mathrm{L}$ $0.1 \%$ solution incubated on the surface for 15 minutes at room temperature, then washed) and subsequently placed into DMEM buffered with $25 \mathrm{mM}$ HEPES (pH 7.0). A suspension containing 200003 T3 cells was introduced into the closed cuvette with a pipette used forced deposition and subsequent spreading was monitored. The inset is a microscope image depicting the spread state characteristic of the cell line.

Figure 4. OWLS cuvette types. a) Image of the underside of a flow-through cuvette with the flow cell and the sealing O-ring. b) Image of a flow-through cuvette with a smaller, ellipsoidal sample volume. c) Image of a flow-through cuvette having an intermediate sized, circular sample volume. d) Image of a septum injector. e) Image of a modified septum injector system. A septum needle has been taken out from its syringe and introduced into a fluid guiding tube to enable continuous washing 
in fluidic systems utilizing a septum cuvette. f) Schematic representation of the arrangement of flowthrough cuvettes.

Figure 5. Estimating the unflushed (dead) volume of a flow-through cuvette. a) Before $t=0 \mathrm{PBS}$ was pumped at $1.4 \mu \mathrm{L} / \mathrm{s}$. At $t=0$ (marked with an upward pointing arrowhead), the flow was changed to a $6 \%$ solution of glycerol in PBS. At $t=1 \mathrm{~min}\left(\right.$ marked with $\left.{ }^{\perp}\right)$ the flow was stopped. At $t=3.5 \mathrm{~min}$ (marked with an arrowhead) flow of glycerol was resumed until $t=10 \mathrm{~min}$, then changed back to PBS. For further explanation see the text.

b) The inverse experiment. Before $t=0$ the cuvette was completely filled with glycerol. At $t=0$ PBS was pumped until the point marked with $\mathrm{T}$. PBS flow was resumed at the next arrowhead. At the end of the circle the cuvette was completely refilled with glycerol again and the same sequence was repeated.

Figure 6. Results of computational fluid dynamic simulations modeling flow in our cuvette (Fig. 4a) at a) low $(1 \mu \mathrm{L} / \mathrm{s})$ and b) at higher $(100 \mu \mathrm{l} / \mathrm{s})$ flow rates. Explanation of colors: the red volume is moving with at least $1 \mathrm{~mm} / \mathrm{s}$, while the blue volume is considered as stationary. The color bar represents the lifetime of streamlines (represented as individual thin lines). Streamlines in the figures suggest that the flow in the cuvette is laminar (there are no currents perpendicular to the direction of flow, nor eddies or swirls of fluid) at a flow rate of $1 \mu \mathrm{l} / \mathrm{s}$, and turbulent at $100 \mu \mathrm{l} / \mathrm{s}$. In the former case, huge volumes remain unflushed in the cuvette (cuvette volumes in blue color, figure a)); acting as dead volumes during sample exchange. Turbulent flow, on the other hand, enables effective sample exchange in the whole cuvette (nearly all blue volumes are eliminated, figure b)). 
Figure 7. The relationship between the tubing length and the time required to saturate the biosensor signal. First, the sample (6\% glycerol solution) was introduced with the SIS-06 injection system through a $20 \mathrm{~cm}$ long tube. Then the effect of tube elongation was investigated using $47,147,447 \mathrm{~cm}$ long tubings. In points marked with „bt”, a bubble trap was integrated in the system, which is equivalent with a tube elongation of $30 \mathrm{~cm}$. Buffer was PBS, and a flow rate of $1.1 \mu \mathrm{L} / \mathrm{s}$ was used in all experiments.

Figure 8. Image of the head of a SIS-06 injector valve: a) inlet for sample introduction, b) screw for setting mode 'Inject' either 'Load', c) tube to downstream fluidics (cuvette), d) calibrated sample loop of the injector, e) tube for waste, $\mathbf{f}$ ) upstream fluidics (bubble trap, pumps). Both the buffer and the limited amount of sample are transferred into the cuvette by continuous pump-driven flow. First the calibrated sample loop has to be filled with the sample while the valve is in the "load" position. The sample loop is disconnected from the flow until the valve is set to the "inject" mode.

Figure 9. a) Operation of an injection valve system. Upward pointing arrowheads indicate the three time points where glycerol solution $(50 \mu 1,5.8 \%)$ was introduced by setting the SIS-06 injection system to 'inject' mode using three flow rates $(1.4,0.7,0.1 \mu \mathrm{L} / \mathrm{s})$. PBS was used as buffer.

b) The ellipsoidal flow-through cuvette could be completely flushed with continously flowing glycerin $(5.8 \% \mathrm{v} / \mathrm{v})$. Upward and downward pointing arrowheads indicate the time points where 5.8\% glycerol solution and MQ were introduced, respectively, with pump-driven flow using flow rates of 1.44 and 0.7 and $0.14 \mu \mathrm{l} / \mathrm{s}$. The volume of the flow cell was approximately $20 \mu 1$. Significantly less amount of solution was needed to saturate the signal if the flow rate was set lower. 
Figure 10. Rearrangement of the fluidic setup (see text) enabled the effects of diffusion on the measurements to be minimized. However, the signal was only saturated if the flow rate was set slow enough $(0.1 \mu \mathrm{l} / \mathrm{s})$. Upward and downward pointing arrowheads indicate the time points where $50 \mu 1$ of glycerol solution (5.8\%) and MQ was introduced, respectively, by setting the SIS-06 injection system to 'inject' mode.

Figure 11 a) Operation of the septum injector. Septum injector was used to introduce either PBS or $6 \%$ glycerol into the OWLS cuvette. Both were introduced manually at the maximum possible rate to eliminate the dead volumes, which seemed to be effective, as second and third injections (marked with additive upward pointing arrowheads) did not further change the signal. b) A septum injector was used to introduce $50 \mu \mathrm{l}$ of $50 \mu \mathrm{g} / \mathrm{ml}$ avidin and its subsequent adsorption was monitored with OWLS. In this original arrangement no continuous flow could be generated, thus succesive injections were needed to characterize the desorption phase.

Figure 12. Experimental usage of the modified septum cuvette-based fluidic system. A septum needle was introduced into the end of a tube; this tube then could be readily introduced into the septum to connect the septum cuvette with a flow reservoir. This system enables a flexible change between two sample introduction strategies: continuous flow and injection of highly restricted sample amounts (Fig. 4e). Figure is adopted from ref. 39. Preceding the online experiment, the OWLS chip underwent an ex situ preparation procedure: its surface was functionalized with polyethylene imine (PEI) and biotinylated with NHS-biotin. The OWLS experiment was initialized by establishing a baseline, then the prepared sensor surface was exposed to avidin by introducing a continuous flow of $50 \mu \mathrm{g} / \mathrm{ml}$ solution through the modified septum cuvette. After a washing step with PBS, the pump generating the analyte flow was stopped and $50 \mu \mathrm{l}$ anti-CRP (C-reactive protein) antibody was 
introduced into the cuvette by manual injection. A $~ 40$ min incubation period was terminated by flushing the cuvette with a continuous flow of PBS. Next, $50 \mu \mathrm{l}$ of $10 \mu \mathrm{g} / \mathrm{ml}$ CRP solution was injected into the cuvette and allowed to bind to the surface for $\sim 20 \mathrm{~min}$. Finally, the cuvette was flushed with a continuous flow of PBS, PBS containing 0.05\% Tween 20, and pure PBS again. For further experimental details, see [39]. 
Figures

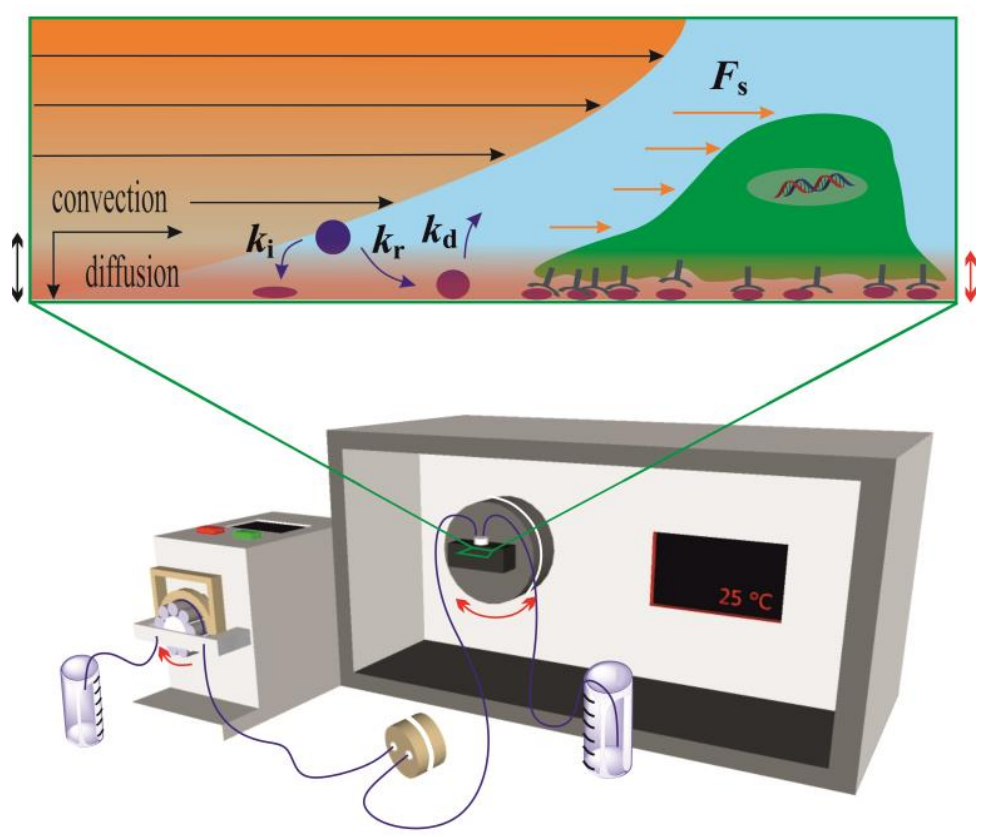

Figure 1 


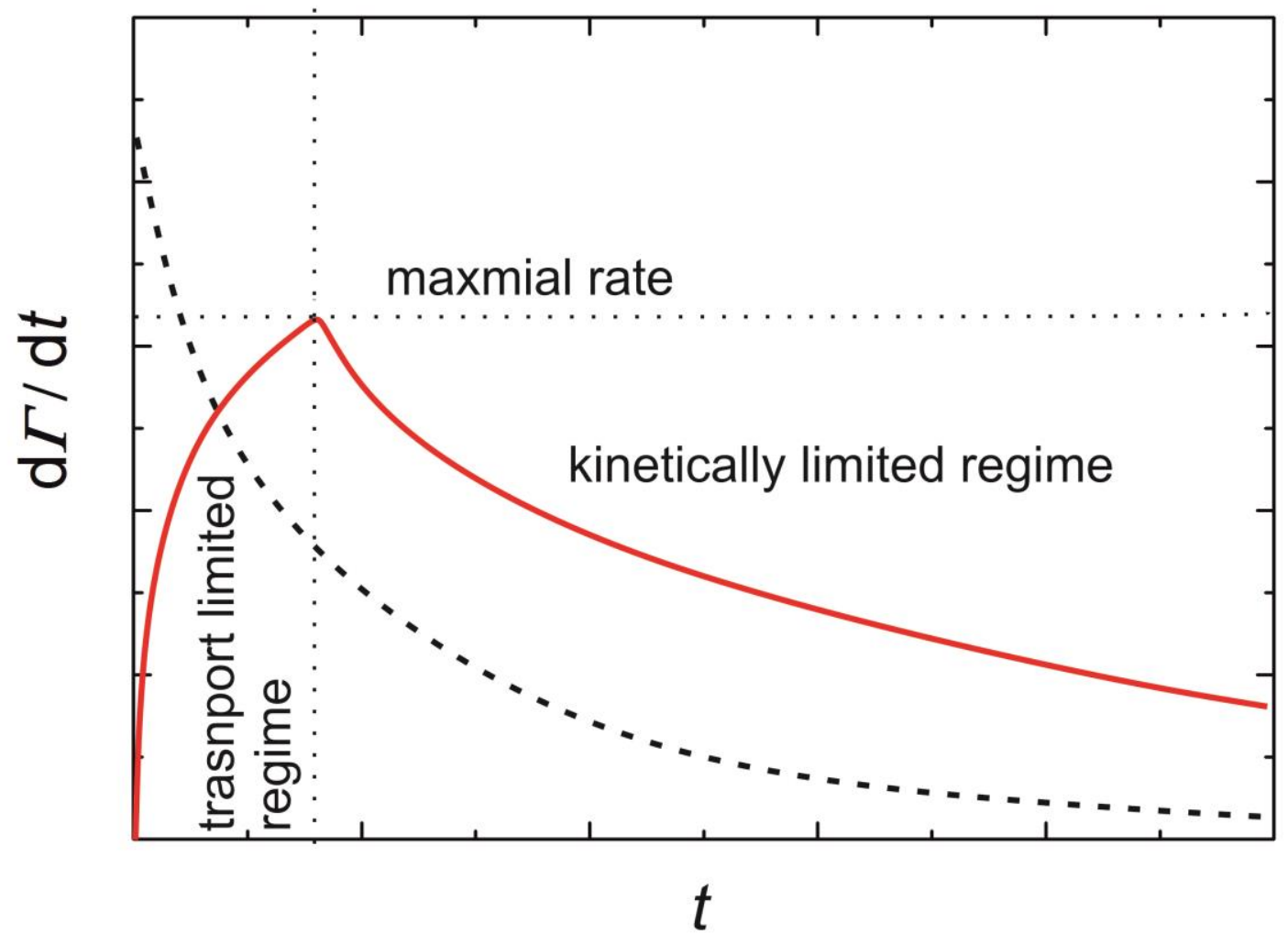

Figure 2 
(a)
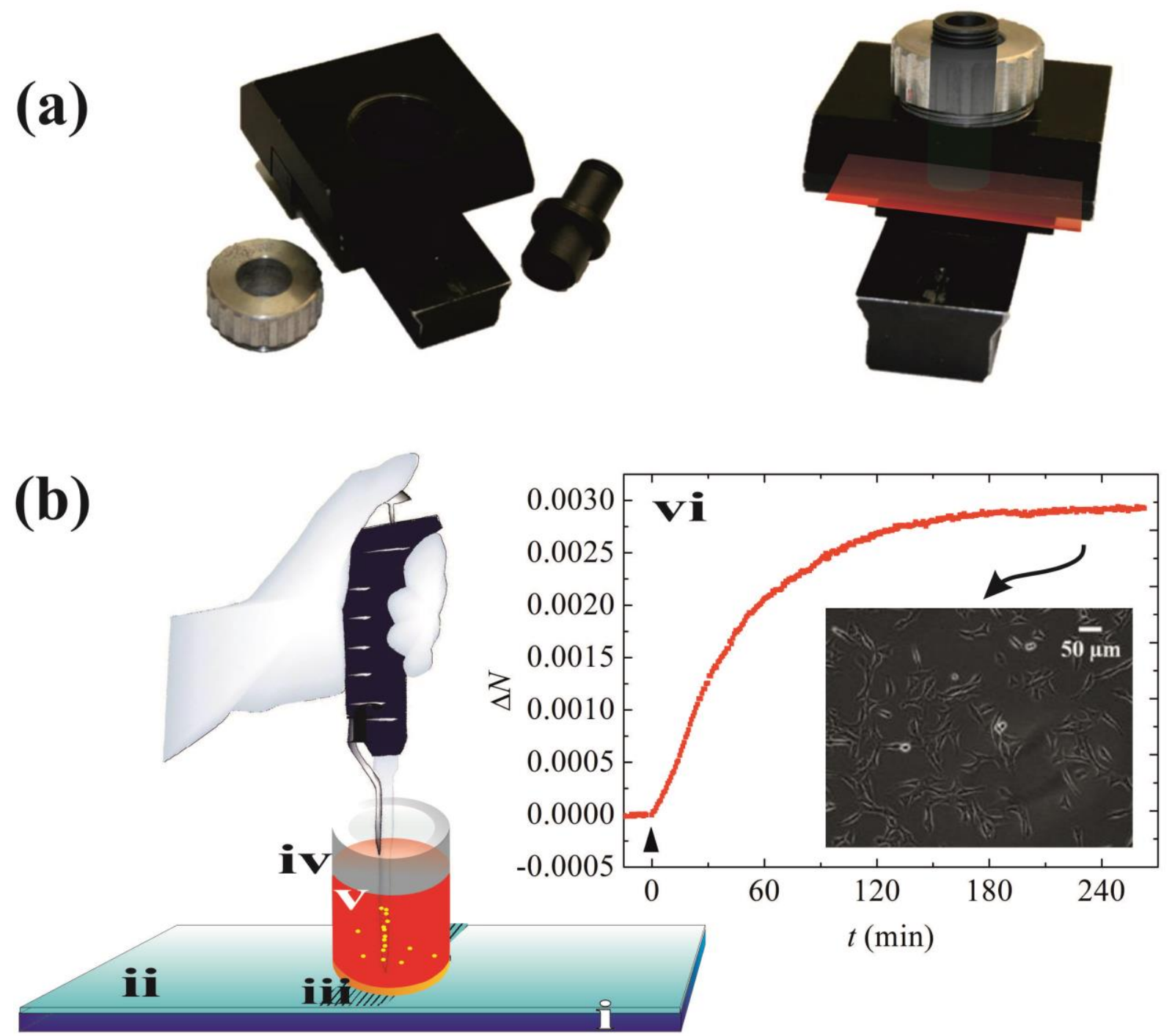

Figure 3 


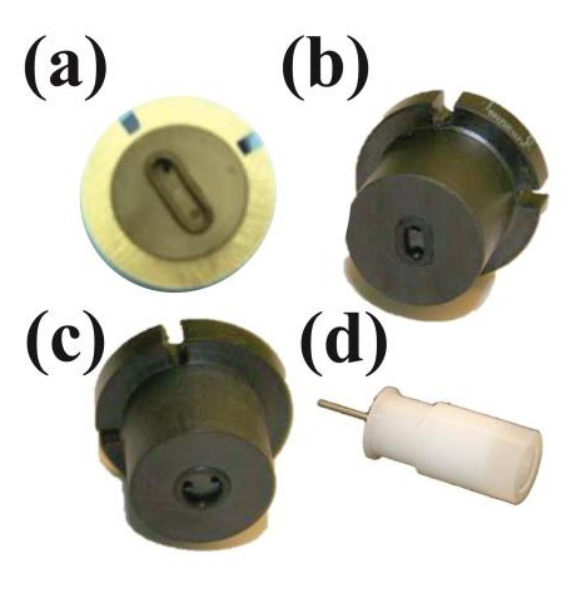

(e) outflow to waste

\section{septum needle placed} in a tube

upstream fluidics: (including pump)
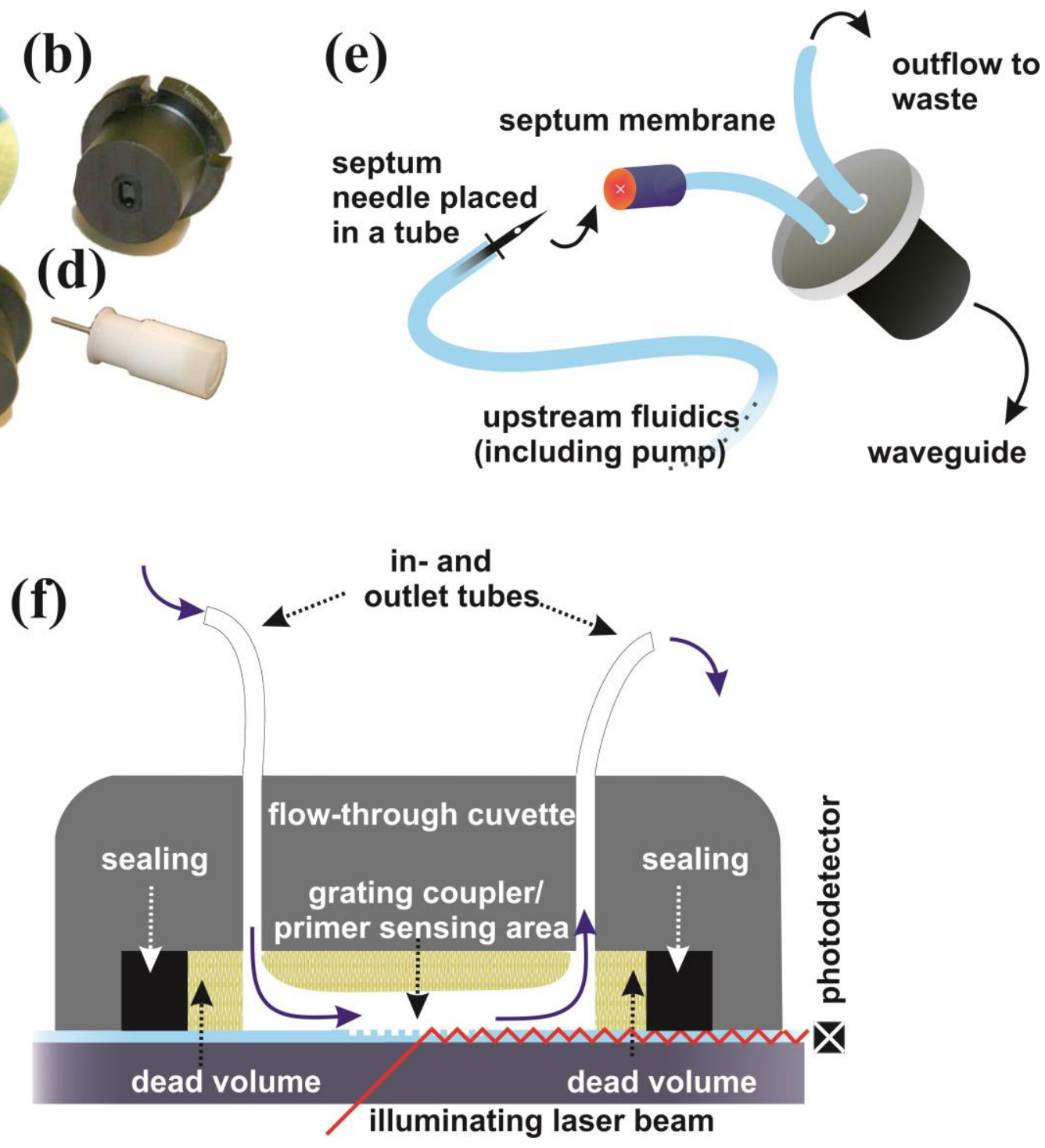

Figure 4. 

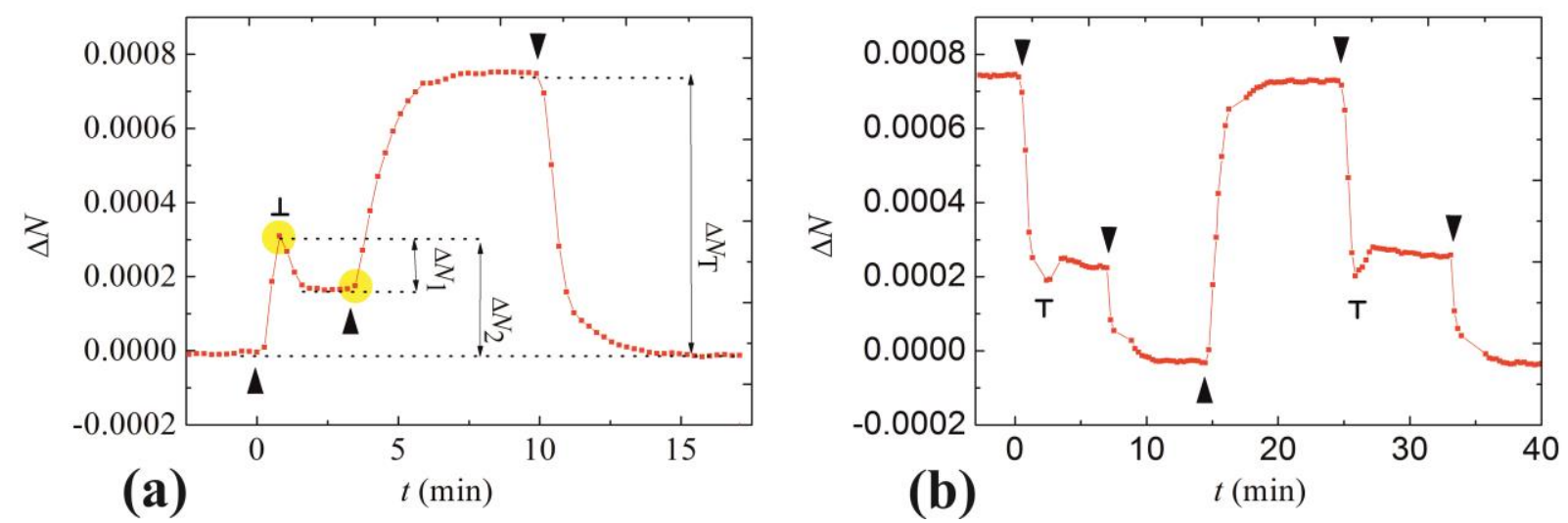

Figure 5 


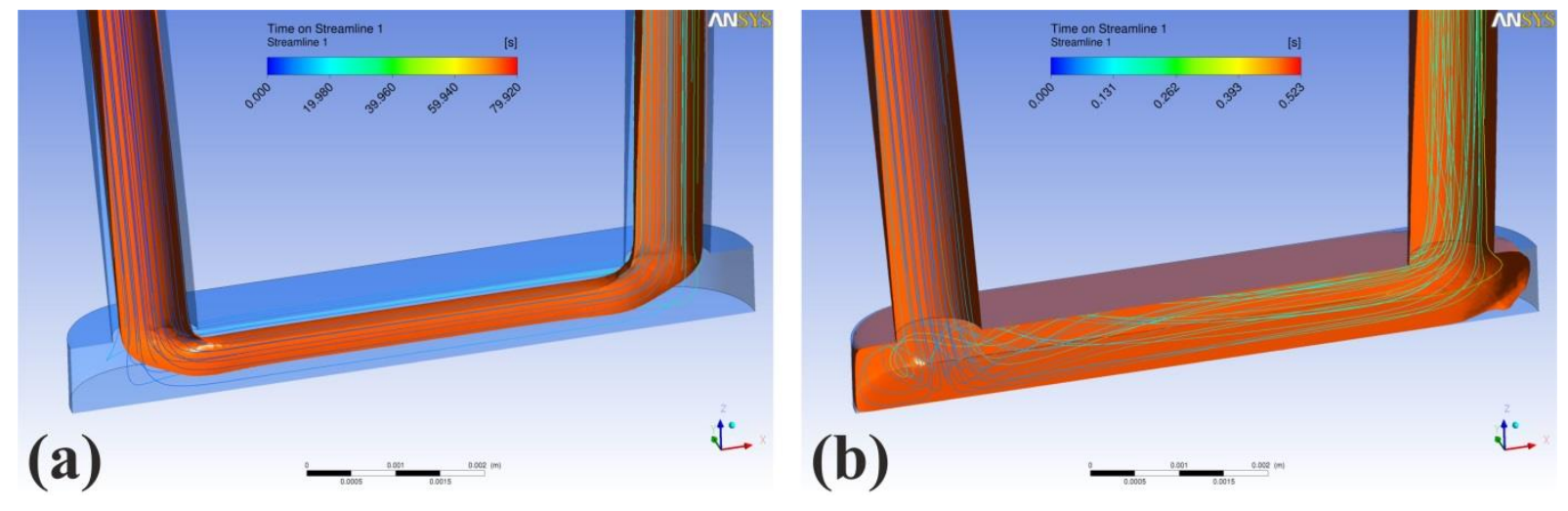

Figure 6 


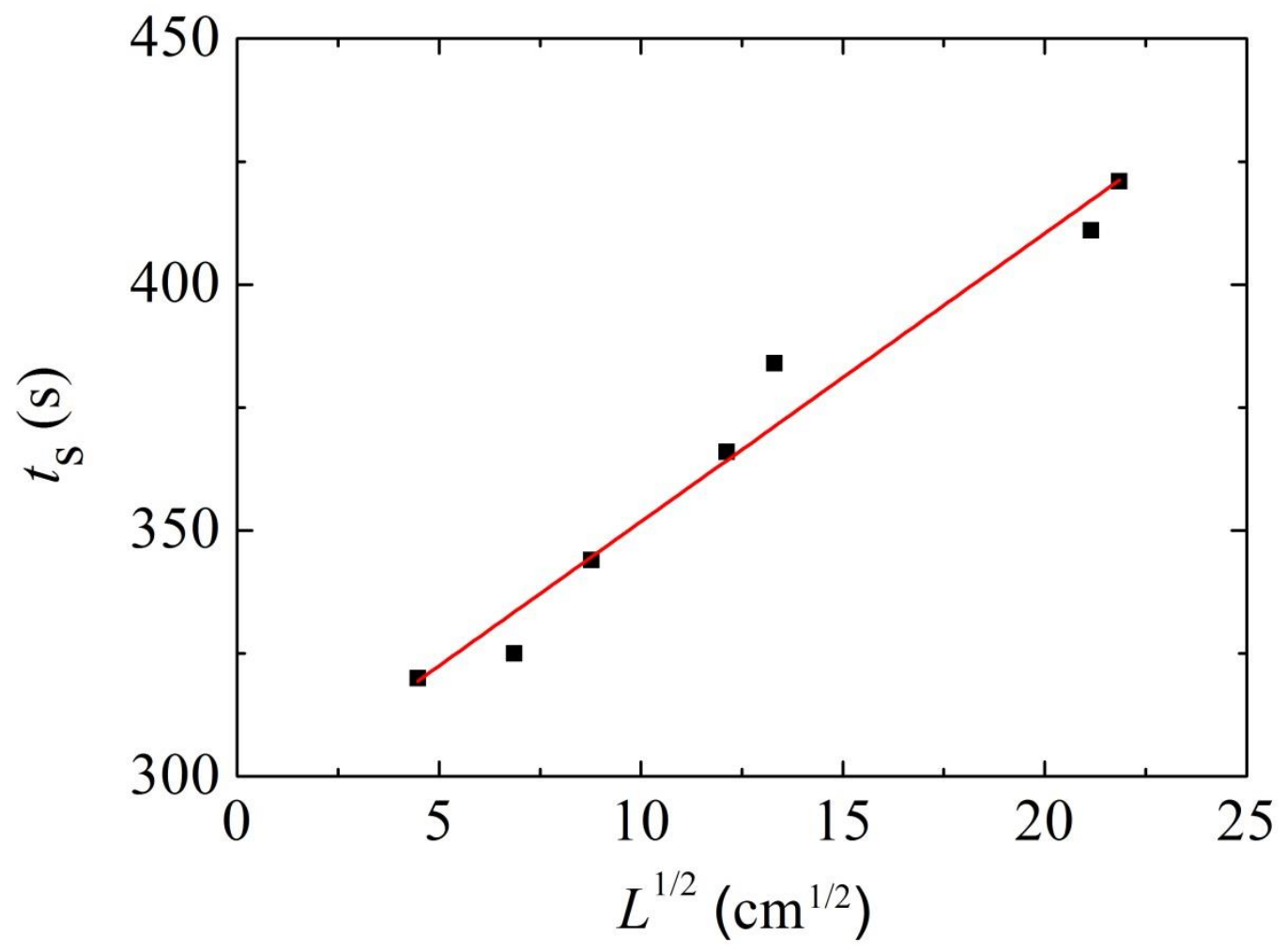




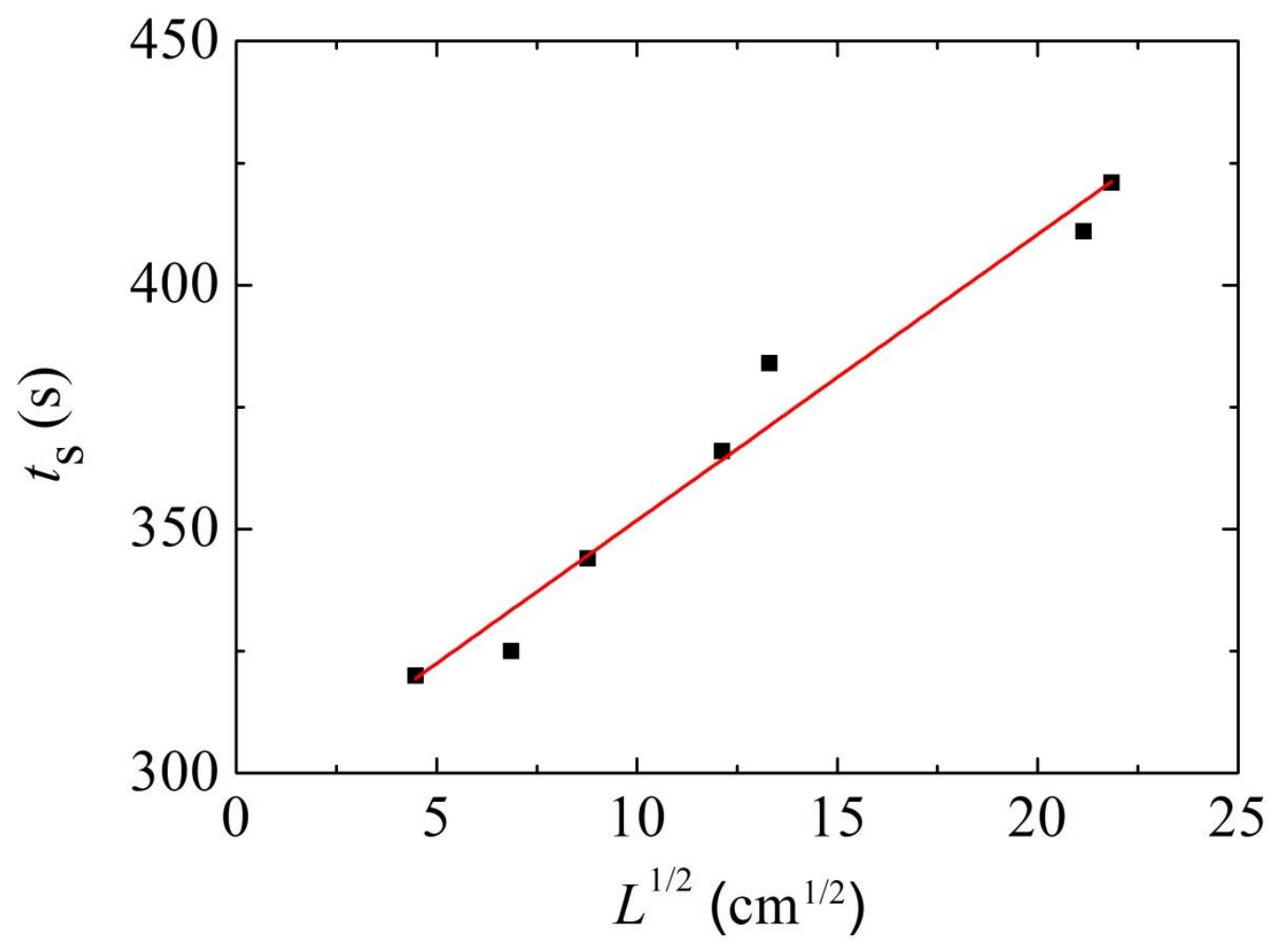

Figure 7 


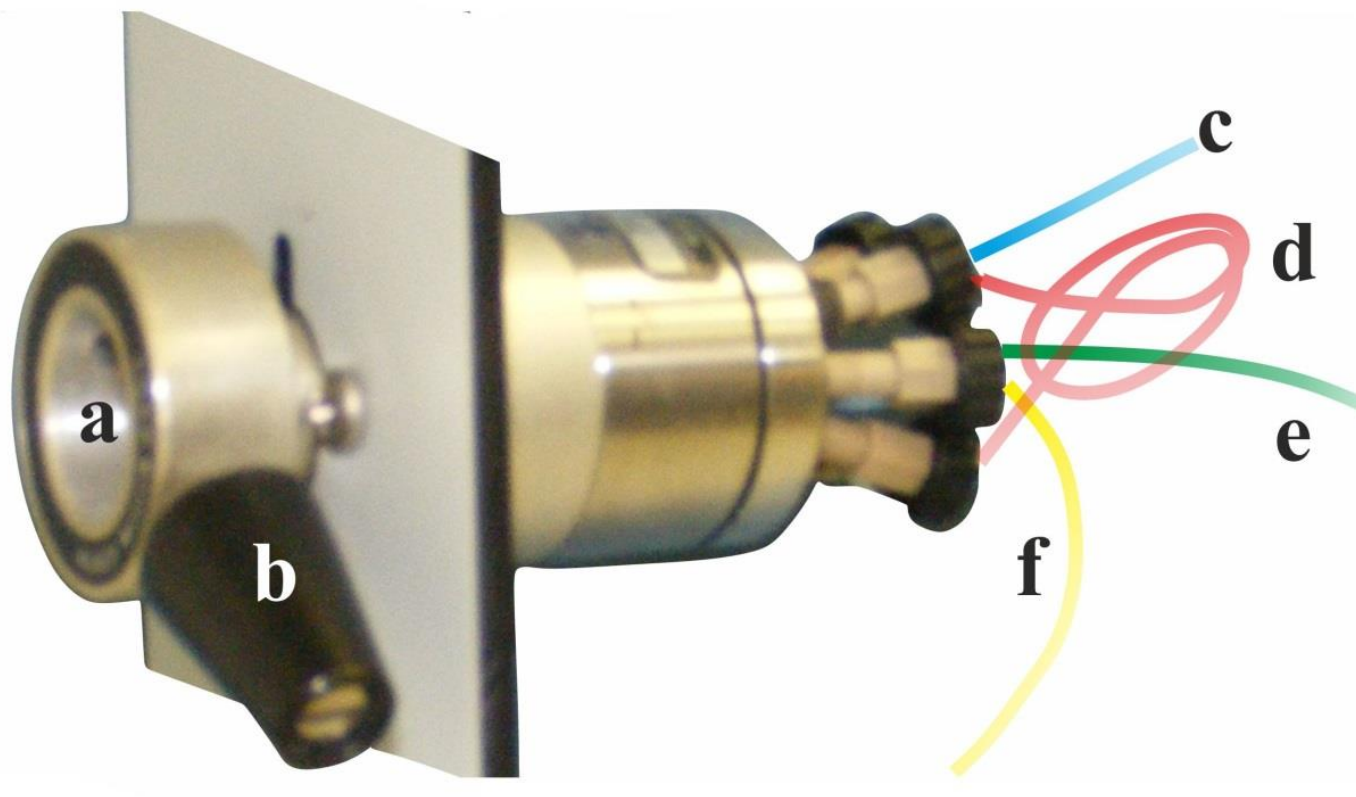

Figure 8 

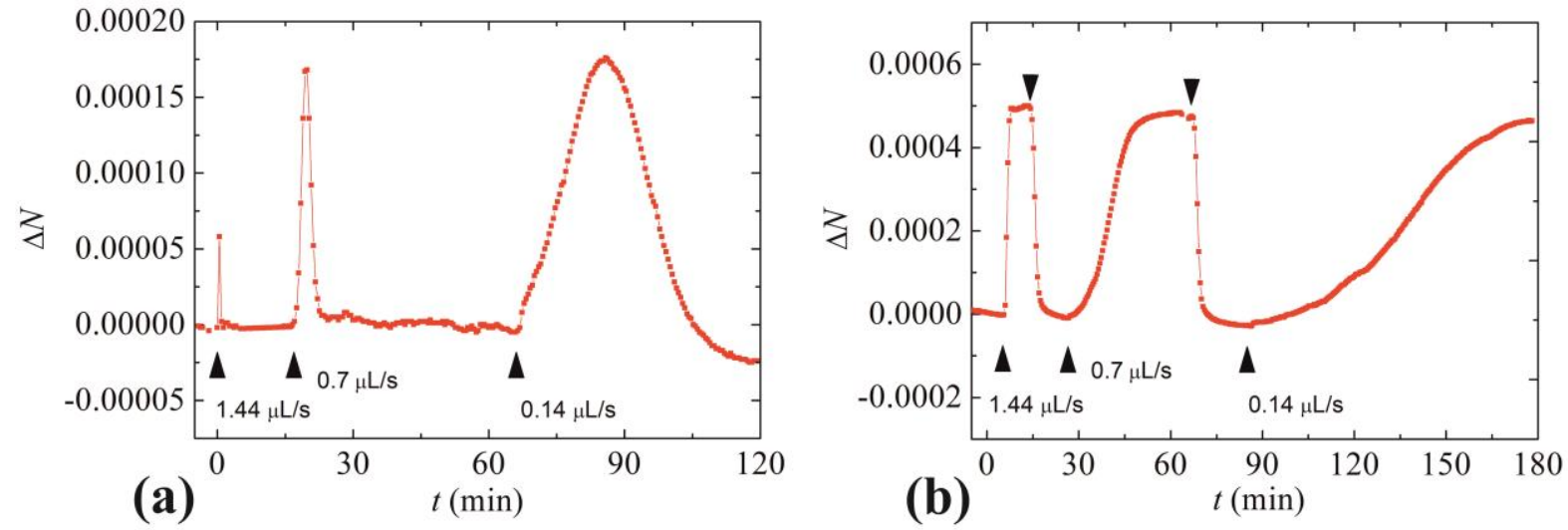

Figure 9 


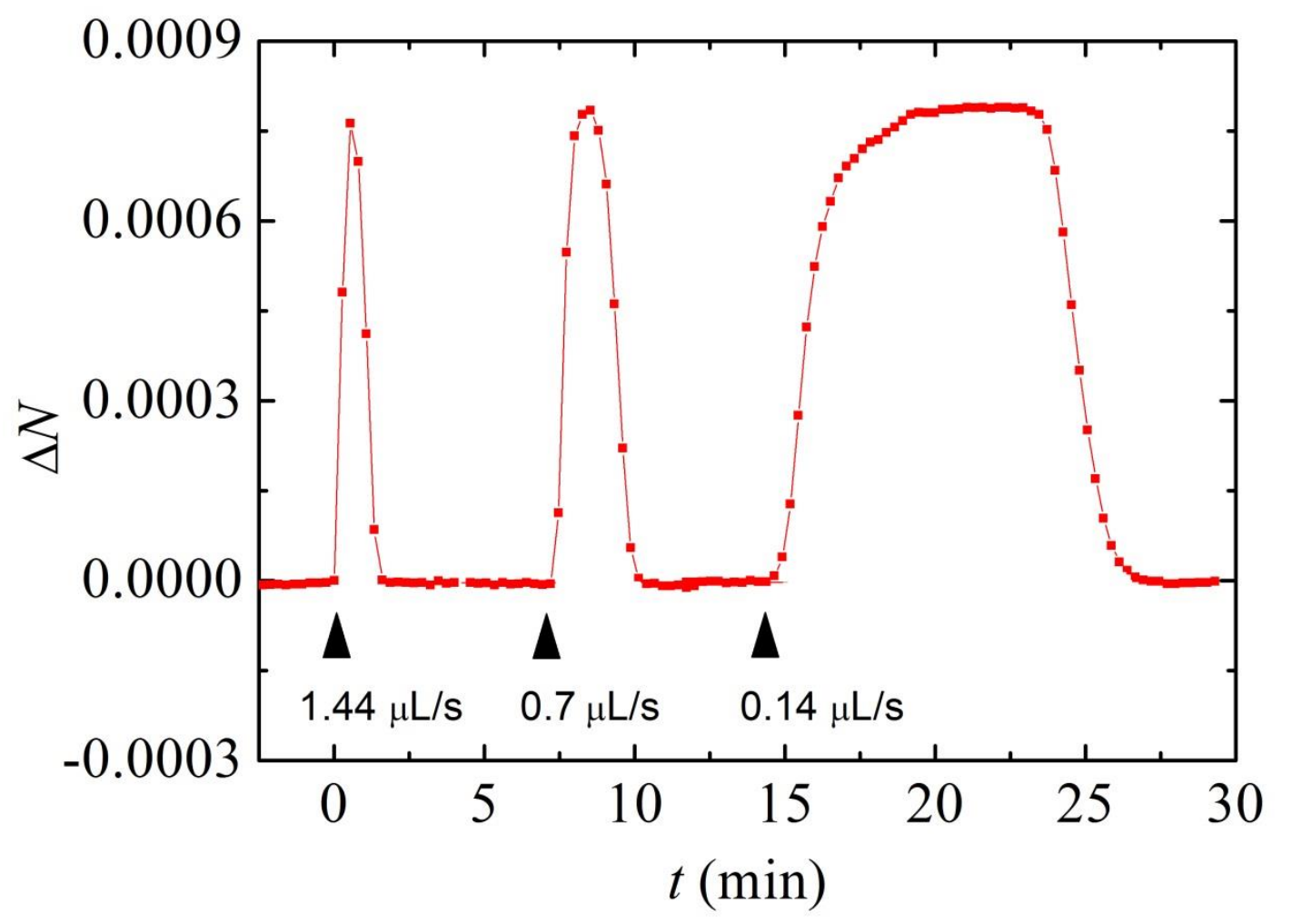

Figure 10 

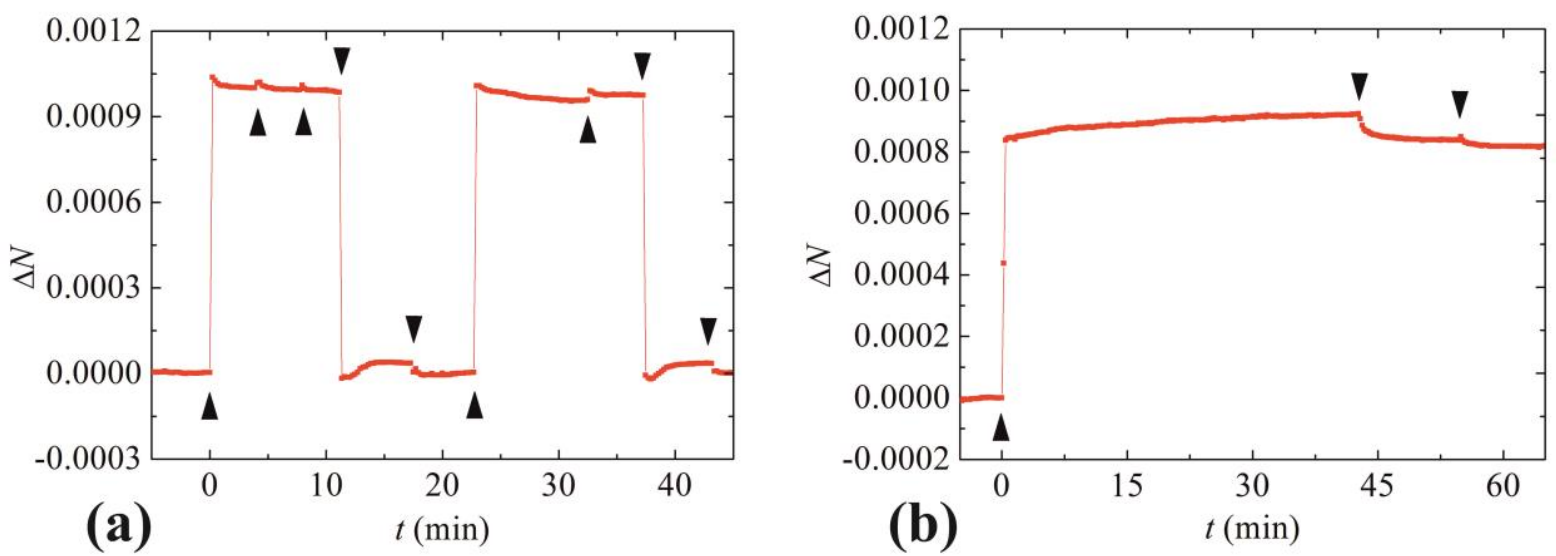

Figure 11 


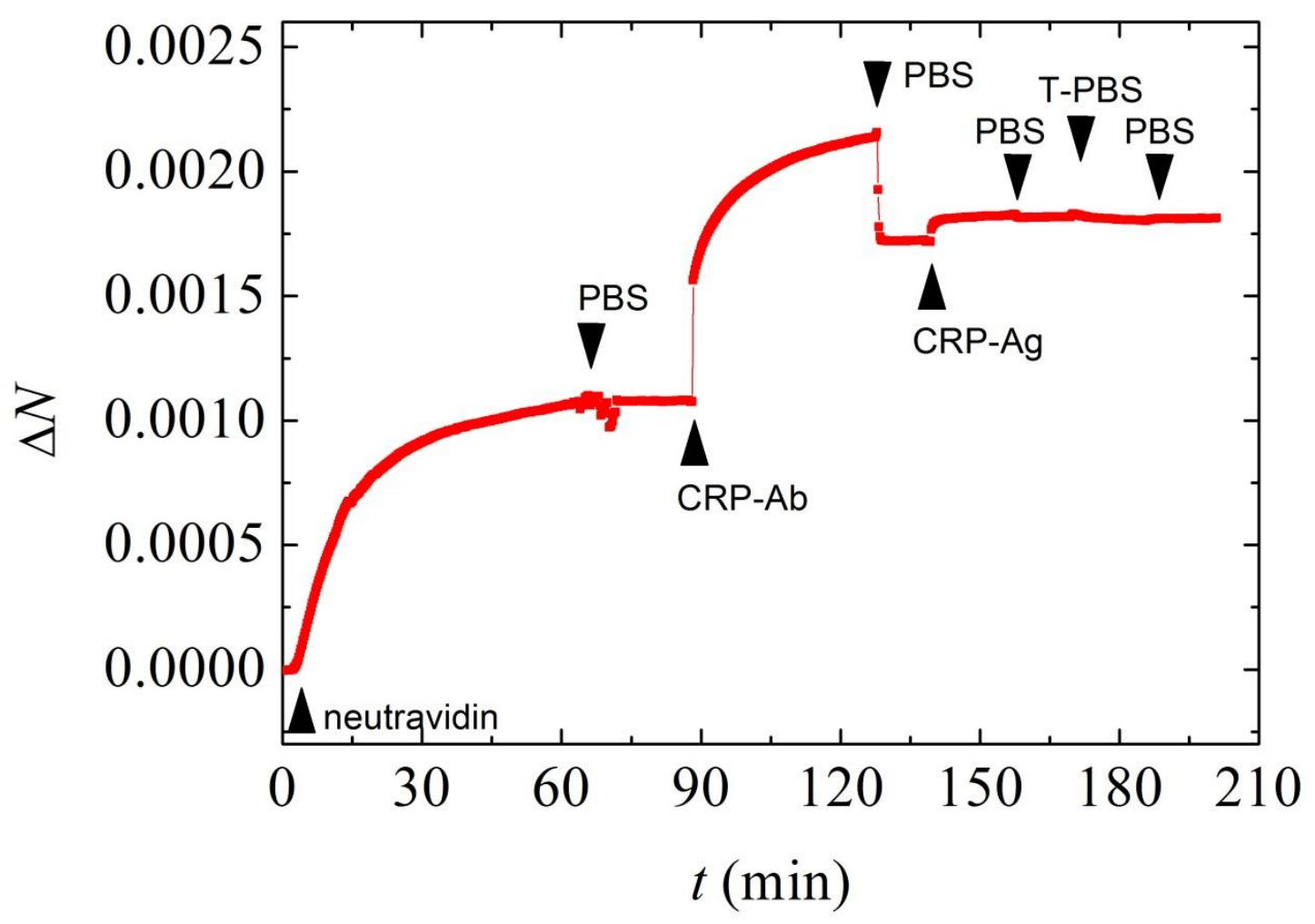

Figure 12 